\title{
À distances raisonnables des structuralismes : logique, langage, formalisation et sciences de l'homme. Une dispute du $20^{\mathrm{e}}$ siècle finissant
}

\author{
Sébastien Plutniak \\ École française de Rome \\ sebastien.plutniak@posteo.net
}

[Version auteur de Sébastien Plutniak (2019), «À distances raisonnables des structuralismes : logique, langage, formalisation et sciences de l'homme. Une dispute du $20 e$ siècle finissant », Zilsel. Science, technique, société, 6, p. 70-115, DOI : 10 . 3917/zil.006.0070.]

À mon père

\section{Table des matières}

1 Une dispute épistémologique
1.1 Quatre itinéraires à proximité puis à distance des structuralismes
1.2 Un différend sur les « usages réglés du rationalisme » en sciences de 1 'homme . . . . . . . . . . . . . . .

2 Les mots et les descriptions en sciences de l'homme

2.1 Une commune limitation du déterminisme linguistique . . . . . .

2.2 Un problème philosophique implicite : descriptions définies et noms

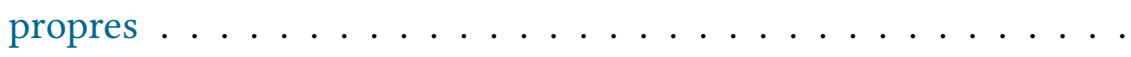

2.3 L'usage des descriptions définies en sciences de l'homme . . . . .

2.4 Les (semi-)noms propres des sciences historiques . . . . . . . .

2.5 Le degré de généralité des concepts employés et ses conséquences sur les raisonnements . . . . . . . . . . . . . . .

3 La spécificité des raisonnements en sciences de l'homme

3.1 Quelle logique pour raisonner sur les phénomènes humains? . .

3.2 Les usages d'une référence commune : la logique naturelle . . . .

3.3 Propositions et énoncés : divergences sur l’analyse des raisonne-

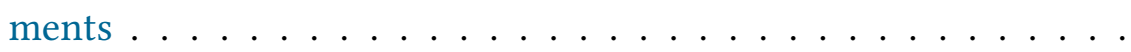

4 Le juste degré de formalisation du langage et des raisonnements
4.1 La formalisation des descriptions
4.2 Les modèles et la formalisation des raisonnements
4.3 Des formalisations qui s'ignorent 
5 Le caractère historique des sciences de l'homme

5.1 Formaliser implique-t-il de dé-historiciser ? . . . . . . . . . . . . .

5.2 Les mécanismes du changement scientifique . . . . . . . . . .

5.3 Pragmatique de l'intervention théorique . . . . . . . . .

L'activité éditoriale concernant le statut et la légitimité des sciences sociales a été particulièrement vive au cours des dernières années. Elle fut notamment marquée par la parution de plusieurs essais à charge contre les sciences sociales ${ }^{1}$ et de réponses - directes ou indirectes - sous la forme de défenses et illustrations, individuelles ${ }^{2}$ ou collectives ${ }^{3}$. Ces publications, destinées à une large audience, ont participé à déplacer la critique publique des sciences sociales au-delà des arènes scientifiques, vers les espaces fortement médiatisés de la «vie intellectuelle ». À ce titre, les positions défendues mirent d'autant plus en avant les rapports entre les dimensions épistémologiques et politiques. La stigmatisation de la « culture de l'excuse » par Manuel Valls et les réactions qu'elle suscita constituèrent un point d'orgue, résumant l'état du statut public accordé en France aux sciences sociales ${ }^{4}$.

Les réponses opposées à ces pourfendeurs des sciences sociales présentent deux caractéristiques notables.

1. D'abord, elles paraissent relever d'un processus de distinction générationnelle, par lequel les auteurs d'une génération ont tendance à privilégier les propositions en rupture avec celles promues par la génération précédente. Les différentes modalités de ce processus, bien connues dans le cas de l'histoire de la sociologie, ont notamment été modélisées par Andrew Abbott ${ }^{5}$.

2. Ensuite, pour ce qui concerne les débats récents, les réponses apportées furent formulées par une génération de chercheurs formés dans le contexte intellectuel vaguement qualifié de poststructuraliste ${ }^{6}$. En rupture avec les travaux structuralistes antérieurs et contre l'antihumanisme, le positivisme et la normativité ayant été associés à ces travaux, ces réponses ont valorisé la thèse d'une spécificité épistémologique des sciences sociales. Ce parti pris, que l'on qualifiera de «différentialiste » implique le refus catégorique de poser le problème dans le cadre d'une épistémologie scientifique qui soit - au niveau le plus général - unifiée ${ }^{7}$.

Cette dynamique générale et ses effets particuliers dans la situation actuelle conduisent ces représentants des sciences sociales à esquiver le cadre du débat intimé par les auteurs mettant en cause la scientificité de leurs travaux. L'espace libéré l'est ainsi au profit des disciplines des sciences naturelles (network science des physiciens, génétique moléculaire des populations humaines, etc.) ou des sciences hu-

1. Bronner et Géhin 2017; Cahuc et Zylberberg 2017.

2. Lahire 2016.

3. Notamment à l'initiative de l'Ehess : les trois volumes publiés en 2012 sous les titres Faire des sciences sociales. Critiquer. Comparer. Généraliser, Paris : Éditions de l'École des hautes études en sciences sociales; et Lemieux 2017.

4. Brandmayr 2018.

5. Abbott 2001.

6. Sur la difficulté de cerner l'objet de cette notion très usitée, quoique différemment selon les régions du monde (aux États-Unis et en France en particulier), cf. Angermuller 2007; Sapiro et Dumont 2016.

7. Existent néanmoins des exceptions, telle que les orientations données dans Joly 2018. 
maines les plus mathématisées (micro-économie, psychologie expérimentale, etc.) jouissant d'un crédit scientifique et d'une reconnaissance politique plus élevés. Ces derniers disposent également aujourd'hui d'un surcroît d'autorité médiatique sur les objets d'enquête qui furent les objets de prédilection des sciences humaines jusqu'au déclin des structuralismes.

Dans ce contexte, où les insuffisances d'une approche différentialiste de l'épistémologie des sciences humaines se manifestent par le recul et la disqualification de leurs discours dans les arènes publiques, je soutiendrai qu'une voie possible de réarmement épistémologique et, partant, d'un potentiel regain de légitimité publique, passe par un retour analytique sur une période à cet égard faste de ces sciences, celle, en l'occurrence, génériquement désignée par le terme de «structuralisme ». Il s'agit, pour reprendre le modèle proposé par Abbott, de neutraliser l'effet de distinction intergénérationnelle en reconsidérant, pour la pratique contemporaine des sciences de l'homme, certains aspects du structuralisme ayant servi de repoussoir à la génération précédente de chercheurs (réintégration qu'Abbott nomme remapping). L'analyse de la dynamique conceptuelle et sociale des sciences de l'homme à partir des rapports intergénérationnels, telle que proposée par Abbott, apporte des éclairages indéniables. Toutefois, comme tout découpage, cette périodisation pose des difficultés analytiques puisque la définition des générations suppose le choix d'un critère parmi des possibilités aussi diverses que l'âge, les relations administratives entre directeurs et doctorants, la période de publication, les filiations intellectuelles déclarées ou non, etc.

L'historiographie des structuralismes et poststructuralismes en sciences de l'homme n'échappe pas à ces difficultés levées par la nécessité de délimiter des générations. Au cours des deux dernières décennies, les travaux à ce propos se sont accrus ${ }^{8}$, ce qui a permis :

- de multiplier les propositions de périodisation ${ }^{9}$;

- de critiquer l'insuffisance des analyses ayant ramené ces courants intellectuels à des «modes »;

- d'insister sur la diversité des réalités rassemblées sous le terme de structuralisme et, plus encore, de poststructuralisme ${ }^{10}$.

Cet article entend contribuer à cette historiographie par l'analyse d'une dispute intellectuelle restée jusqu'ici relativement inaperçue, bien qu'elle ait porté sur des problèmes fondamentaux d'épistémologie des sciences de l'homme, cruciaux pour l'approche des démarcations réalisées entre structuralismes et poststructuralismes. Elle opposa principalement deux praticiens et théoriciens des sciences humaines, le sociologue Jean-Claude Passeron (1930-) et l'archéologue, linguiste, et spécialiste de documentation Jean-Claude Gardin (1925-2013). Les positions qu'ils ont respectivement défendues ont impliqué plus particulièrement les travaux de deux autres chercheurs contemporains, le philosophe Gilles-Gaston Granger (1920-2016) et le logicien Jean-Blaise Grize (1922-2013). À travers l'analyse des idées ${ }^{11}$ en conflit dans cette dispute ${ }^{12}$, deux enjeux historiographiques parti-

8. Léon 2013.

9. Dosse 1991 ; Dosse 1992 ; Milner 2002.

10. Puech 2013; Angermuller 2007; Bert et Lamy 2016.

11. L'histoire des idées, parfois dépréciée, bénéficie d'une longue tradition d'études, tant pratiques que théoriques. $C f$. Mandelbaum 1965; Skinner 1969.

12. L'analyse des disputes intellectuelles, centrée sur les réseaux notionnels, se distingue de celle 
culiers seront abordés. Le premier concerne les distinctions générationnelles évoquées précédemment : dans le cas des structuralismes et poststructuralismes, cette dispute permet de révéler le détail de constructions conceptuelles complexes qui ne recouvrent pas nécessairement des définitions entièrement distinctes et exclusives de telles générations intellectuelles. L'enjeu de cette dispute ayant aussi été de délimiter les conditions d'exercice légitimes des sciences de l'homme - ce qui a des conséquences sociales et normatives sur les pratiques - un second enjeu concerne les manières dont ces auteurs ont endossé le rôle de théoricien dans ce domaine scientifique. Leurs « interventions épistémologiques » seront aussi considérées comme des actes de positionnement dans un champ intellectuel. Compte tenu de ces enjeux, cette dispute présente trois caractéristiques intéressantes.

D’abord, concernant les problèmes de périodisation générationnelle, elle correspond, sur le plan intellectuel, à une discontinuité générationnelle (entre structuralismes et poststructuralismes). Toutefois, sur le plan sociologique, elle correspond à une continuité générationnelle, puisque ce sont bien les mêmes quatre auteurs qui agissent au cours de la période examinée. Leurs parcours ont en commun de comporter une première phase à proximité des pratiques et des milieux structuralistes, laquelle comporta (Gardin, Granger), ou a été suivie par (Passeron, Grize), un repositionnement à distance raisonnable de la forme particulière de structuralisme à laquelle chacun d'entre eux s'était familiarisé. Ainsi, ces chercheurs n'ont pas déclaré de rupture radicale avec les propositions théoriques antérieures et leurs promoteurs, bien qu'il s'agisse d'un des procédés fréquents de fondation en sciences humaines.

Ensuite, ce débat a été conduit à distance des lieux susceptibles de conduire aux requalifications des approches structuralistes en tant que «mode » ou que « courant intellectuel »: premièrement, dans la mesure où il a été mené dans des arènes spécialisées, par des auteurs peu soucieux de «faire école » ou d'obtenir des reconnaissances en dehors de ces arènes (de type médiatique, politique, etc.); deuxièmement, par la nature épistémologiquement exigeante et particulièrement technique de cette dispute.

Enfin, les épistémologies respectivement défendues par Gardin et Passeron, quoique opposées, partageaient néanmoins l'objectif de définir positivement la scientificité des sciences de l'homme. Elles se sont ainsi distinguées des critiques les plus radicales exprimées par les travaux dits poststructuralistes (les post modernismes et les différents « tournants » : pragmatique, linguistique, microhistorique, etc.) en se refusant à rabattre entièrement les sciences de l'homme sur la littérature ou sur l'action politique.

Cette dispute revêt un intérêt supplémentaire puisque le livre Le raisonnement sociologique ${ }^{13}$ de Passeron a été fondamental pour les tentatives de réédification des sciences sociales sur une épistémologie qui leur soit propre. Trois observations permettent de mesurer l'importance et les effets de ce livre : le nombre de citations dont il a fait l'objet - de l'ordre de $140{ }^{14}$; le fait qu'une part (ici non quantifiée) de ces citations prenne la forme d'argument d'autorité, jugées suffisantes

des controverses, qui porte tout autant sur les objets, les personnes et leur autorité; $c f$. Fabiani 2007, p. 52 .

13. Passeron 1991.

14. D’après Google Scholars, consulté en juillet 2019. 
pour avancer la thèse d'une spécificité épistémologique des sciences sociales; le nombre important de traductions dont il a fait l'objet (en portugais, vietnamien, espagnol, et anglais). Ce livre étant au cœur de la dispute entre Passeron et Gardin, elle fait de cette dernière une entrée pertinente pour aborder les profondes transformations des sciences de l'homme et de leur théorie à la fin du $20^{\mathrm{e}}$ siècle, période marquée par l'effondrement des «grands systèmes » (structuralisme, marxisme) et la généralisation de l'informatique.

Il s'agit donc d'analyser cette dispute en ce qu'elle révèle la manière dont les démarcations entre structuralismes et poststructuralismes ont pu réellement être opérées dans le cadre technique d'une dispute épistémologique, laquelle fut - à bien des égards - exceptionnelle dans la littérature francophone.

Cette étude, principalement fondée sur les publications et secondairement sur des documents d'archives ${ }^{15}$, prolonge une première analyse de cette dispute ayant abordé les aspects relatifs à l'épistémologie de l'archéologie ${ }^{16}$. La présente contribution s'ouvrira par une présentation des acteurs de la dispute, de sa chronologie et des principales thèses ayant été opposées. Les parties suivantes analyseront les clivages les plus saillants des thèses défendues par les auteurs.

- Je commencerai par circonscrire les termes permettant d'établir les descriptions des phénomènes humains, avant tout raisonnement à leur propos.

- Puis, je détaillerai les raisonnements employés, en tenant compte de l'éventuelle existence d'un type de raisonnement spécifique aux sciences de l'homme.

- J'analyserai ensuite la formalisation des descriptions et des raisonnements, laquelle implique un certain degré d'abstraction par rapport à leurs ancrages historiques et soulève le problème de la fixation de ce degré.

- Il sera enfin question du caractère historique des raisonnements menés en sciences de l'homme et des conséquences de cette propriété pour leur communication et leur validation.

\section{Une dispute épistémologique}

\subsection{Quatre itinéraires à proximité puis à distance des structuralismes}

Les quatre acteurs de cette dispute, à savoir, Gardin et Passeron en première ligne, Granger et Grize en arrière-garde, eurent des parcours intellectuels en partie similaires. Tous s'impliquèrent premièrement dans des travaux menés auprès d'auteurs centraux d'une forme ou d'une autre de structuralisme, puis développèrent ensuite des programmes de recherche à distance de ces orientations structuralistes.

Formé en économie, en linguistique et en langues orientales, Gardin a participé, à partir de 1955, comme auditeur et orateur, au séminaire «Religions comparées des peuples sans écriture » de Claude Lévi-Strauss (1908-2009) à l'Ephe ${ }^{17}$. Devenu archéologue et alors plongé dans ses premières expérimentations d'automatique

15. Fonds J.-C. Gardin de la Maison Archéologie \& Ethnologie à Nanterre (abrégé MAE); fonds J.-C. Gardin de la Bibliothèque nationale et universitaire de Strasbourg (BNU); fonds C. Lévi-Strauss du Laboratoire d'anthropologie sociale, Paris (LAS).

16. Plutniak $2017 \mathrm{~b}$.

17. Plutniak 2018, p. 13-14. 
documentaire, il initia plusieurs projets avec Lévi-Strauss et ses collaborateurs, dont l'analyse structurale des mythes d'origine des Indiens Zuni. En 1960, LéviStrauss le chargea d'un séminaire intitulé « Méthode moderne et révolution dans les techniques des sciences humaines ». Puis, en 1962, après voir été recruté à la $6^{\mathrm{e}}$ section de l'Ephe, Gardin donna les premiers enseignements liés à sa propre chaire, intitulée «Sémiologie et documentation».

$\mathrm{Au}$ cours des décennies suivantes, il poursuivit ses divers programmes de recherches tant en automatique documentaire, en linguistique qu'en archéologie. Bien que ses orientations théoriques et méthodologiques se sont éloignées de celles de Lévi-Strauss - notamment quant à l'usage des mathématiques -, Gardin conserva à son égard une reconnaissance et une estime intellectuelle intacte, comme en atteste leur correspondance. En 1968, Gardin lui adressa ainsi la copie d'un article dans lequel il établissait des différences entre l'analyse documentaire qu'il pratiquait et l'analyse structurale ${ }^{18}$. Lévi-Strauss lui répondit très favorablement :

«[...] cela me donne l'occasion de vous remercier de l'excellent article dont vous venez de m'envoyer la photocopie et avec lequel je me sens pleinement d'accord. Moi aussi je commence à être excédé de ce que j'appelle dans l'intimité "le structuralisme-fiction" $» .{ }^{19}$

Passeron, quant à lui, a suivi une formation philosophique à l'École normale supérieure de la rue d'Ulm, à Paris. Au cours des années 1960, il participa aux travaux animés par Pierre Bourdieu (1930-2002) qui s'inscrivaient alors dans une forme de structuralisme en sociologie ${ }^{20}$. Ensemble, Passeron et Bourdieu ont signé plusieurs enquêtes aujourd'hui célèbres, dont Les héritiers (1964) et La reproduction (1971). Les orientations épistémologiques de ces travaux ont été synthétisées dans Le métier de sociologue ${ }^{21}$. Au cours de la décennie suivante, Passeron prit ses distances avec ces orientations. Il s'attacha à établir d'autres fondations épistémologiques qui firent l'objet de sa thèse d'État défendue en $1980^{22}$. En quittant, en 1982, l'université de Paris 8 pour rejoindre l'EHEss et fonder un laboratoire à Marseille, il réunit les moyens nécessaires à l'approfondissement de ce nouveau programme épistémologique ${ }^{23}$.

Granger, également normalien de la rue d'Ulm, a été formé en philosophie et en mathématiques. Il consacra ses premiers travaux à la philosophie des sciences de l'homme, et en particulier aux mathématiques sociales. Dans le contexte intellectuel parisien, la philosophie des sciences était alors dominée par l'approche structuraliste de Martial Gueroult (1891-1976), centrée sur la seule structure conceptuelle interne d'une œuvre. Son étude de l'œuvre de Spinoza constituait alors l'exemple paradigmatique de cette approche. En faisant paraître Pensée formelle et sciences de l'homme ${ }^{24}$ en 1960, Granger opéra une double prise de distance : d'une

\footnotetext{
18. Gardin 1965.

19. Lettre de C. Lévi-Strauss à J.-C. Gardin, 25-04-1968, LAs, fr/cdf/las/FLAS.A.

20. Défendue par Jean-Louis Fabiani (Fabiani 2016), l'interprétation du rapport de Bourdieu au structuralisme fait néanmoins débat. Voir la critique de Joly 2018.

21. Bourdieu et al. 1969.

22. Passeron 1980.

23. Des éléments biographiques plus complets figurent dans l'introduction de la version espagnole du Raisonnement sociologique (Moreno Pestaña 2011) et dans deux entretiens (Passeron et al. 1996; Costey et Fossier 2009).

24. Granger 1960.
} 
part, par rapport à l'approche structuraliste de Gueroult en réintroduisant des facteurs externes à l'architecture conceptuelle des œuvres philosophiques, dont les apports des sciences; et, d'autre part, par rapport aux travaux structuralistes contemporains en sciences de l'homme, en tempérant les engouements formalisateurs qui caractérisaient alors certains d'entre eux.

Grize a été initié aux mathématiques et à la logique. De 1958 à 1968, il travailla au Centre international d'épistémologie génétique à Genève, auprès de Jean Piaget (1896-1980), avec qui il réalisa plusieurs publications. Scientifique prolifique, Piaget est l'auteur notable de l'une des premières synthèses sur le structuralisme ${ }^{25}$. En 1969, Grize quittait Genève afin de fonder puis diriger un Centre de recherches sémiologiques à Neuchâtel. Il y développa un programme de recherche sur la logique naturelle, se démarquant alors de la logique piagétienne et plus largement de la logique standard. La logique naturelle concerne les schématisations construites à l'aide de langues naturelles. Ces schématisations se distinguent des démonstrations - dont traite la logique standard - par le fait d'être le résultat d'un comportement, d'un processus de communication impliquant un énonciateur, ce qui limite la possibilité de les formaliser ${ }^{26}$.

Les références et usages respectifs que ces quatre auteurs ont établis entre leurs travaux se sont inscrits dans des itinéraires intellectuels similaires dans leurs grandes scansions, mais aussi dans leur attachement au rationalisme scientifique, quoique selon des modalités différentes.

\subsection{Un différend sur les « usages réglés du rationalisme» en sciences de l'homme}

Passeron, Gardin, Granger et Grize se distinguent par la forme particulière de structuralisme dont ils se sont rapprochés puis distancés. Pour autant, ces distances peuvent être qualifiées de « raisonnables » en ceci que les critiques développées par ces quatre chercheurs conservent pour assise des valeurs épistémiques réalistes et rationalistes, alors même que celles-ci étaient vigoureusement remises en cause par les tenants des divers poststructuralismes. Ainsi, l'affirmation suivante de Passeron aurait probablement fait consensus entre Gardin, Granger et Grize :

« Décréter l'Aufklärung béotien ou criminogène est aujourd'hui le plus court chemin pour régler leur compte, sans avoir à les affronter, aux usages réglés ou circonstanciés du rationalisme dans les sciences et l'histoire comme dans la morale et la politique. ${ }^{27}$

C'est toutefois sur les modalités de la régulation en question que Gardin, en particulier, se démarquait radicalement. Ainsi, au-delà de l'accord minimal entre les quatre auteurs, leurs divergences sur les directions et les modalités de ces « distances raisonnables » ont été au cœur de la dispute alimentée par Passeron et Gardin de 1980 aux années 2010.

On retiendra ici trois principaux jalons dans le déroulement de cette dispute. En 1991, Passeron fit paraître le Raisonnement sociologique, ouvrage rassemblant

\footnotetext{
25. Piaget 1968.

26. Grize 1993; Grize 1994a, p. 282.

27. Passeron 2002, p. 181.
} 
diverses études antérieures, ainsi qu'une systématisation des considérations épistémologiques développées dans son doctorat d'État de 1980. Dans ce livre, il fustigeait les ambitions que poursuivait Gardin dans ses travaux collectifs sur les systèmes experts.

En 1993, ce dernier publiait une critique des «Propositions et scolies » qui clôturaient l'ouvrage de Passeron ${ }^{28}$. Aux yeux de Gardin, ces propositions constituaient plus généralement une manifestation particulière de la thèse qu'il nommait génériquement thèse de la «troisième voie ». Il désignait ainsi les approches défendant la possibilité d'un régime épistémologique médian entre science et littérature $^{29}$. Passeron défendait une variante de cette thèse, depuis la sociologie, tout en étendant sa portée et sa validité à ce qu'il nommait les « sciences historiques » (dont le noyau serait constitué par la sociologie, l'histoire et l'anthropologie) ${ }^{30}$. Puisant au dualisme méthodologique défendu par Max Weber, il fondait ses arguments sur la spécificité de ce qu'il nommait et caractérisait comme « raisonnement historique », lequel serait propre aux « sciences historiques », c'est-à-dire principalement la sociologie, l'histoire et l'anthropologie. Contrairement à la sociologie, l'archéologie - discipline dont se réclamait Gardin - avait alors récemment commencé à acquérir des moyens d'autonomie disciplinaire par rapport à l'histoire, l'histoire de l'art, et la philologie. Cette disciplinarisation s'est accompagnée, en France, d'une timide production d'écrits théoriques. Gardin a été (et reste) l'un des rares contributeurs francophones ${ }^{31}$ dans ce domaine, quoique ses intérêts intellectuels allaient bien au-delà de cette seule discipline.

En 2006, dans l' « Avertissement » qui ouvrait la seconde édition de son livre, Passeron a souligné le caractère fondamental des critiques de Gardin. Un examen minutieux de cette seconde édition conduit toutefois à constater qu'il n'a introduit aucune modification dans les passages concernés, à l'exception du sous-titre de l'ouvrage. Durant une vingtaine d'années et parallèlement à ces trois publications, Gardin et Passeron ont alimenté leur différend dans nombre de leurs publications ${ }^{32}$.

La critique de Gardin de 1993 peut être réduite à sept arguments principaux, concernant : 1) le statut des épreuves empiriques, 2) la structure logique des théories, 3) la spécificité des raisonnements en sciences humaines, 4) la formalisation en sciences humaines, 5) les critères de décision entre descriptions différentes, 6) les critères de décision entre théories contradictoires, 7) la distinction entre langages formels et langages naturels. Compte tenu du haut degré de généralité des arguments opposés dans cette dispute, chacun d'entre eux pourrait nécessiter le recours à une abondante bibliographie de philosophie des sciences. Cet article, qui ne prétend pas livrer une analyse exhaustive, ne portera que sur un nombre limité d'aspects, progressant depuis les plus abstraits vers les plus pratiques ${ }^{33}$. Le pre-

\footnotetext{
28. Gardin 1993.

29. Pour une autre illustration fameuse d'une telle thèse, voir : Lepenies 1990.

30. Dans la suite de ce texte, on emploiera «sciences historiques » pour faire référence à l'acception de Passeron et, indifféremment, «sciences humaines » et «sciences de l'homme » pour désigner généralement les sciences relatives à l'homme.

31. Gardin 1979.

32. Pour le détail et la chronologie des lieux et des publications liés à cette dispute, $c f$. Plutniak 2017b, p. 10-12.

33. Sans être négligée, la chronologie de cette dispute ne sera donc pas le mode d'exposition
} 
mier concernera un aspect antérieur à l'exercice des raisonnements, à savoir les termes qui y sont employés en sciences de l'homme afin d'établir des descriptions de l'état du monde.

\section{Les mots et les descriptions en sciences de l'homme}

\subsection{Une commune limitation du déterminisme linguistique}

Actifs scientifiquement depuis les années 1950, Passeron et Gardin étaient familiers d'une ambiance intellectuelle dominée par la linguistique. Ultérieurement, lors de leur dispute, l'un et l'autre se sont accordés sur le fait de dénier à cette science le rôle prépondérant qui lui était accordé dans l'analyse des raisonnements, tant en épistémologie qu'en intelligence artificielle. Passeron insistait sur le fait qu'en se focalisant sur le langage, l'analyse reste aveugle aux caractéristiques et aux difficultés spécifiques de l'observation des actions sociales, lesquelles sont nécessairement historiquement situées dans des « contextes » qui comportent davantage que des faits linguistiques ${ }^{34}$.

Gardin, quant à lui, distinguait et admettait une certaine autonomie entre la dimension cognitive et les expressions linguistiques. Depuis les « codes descriptifs » qu'il réalisa durant les années 1950 jusqu'aux applications de la méthode de réécriture logiciste des années 1990, il s'attacha à diversifier les objets sur lesquels portaient ses expérimentations (textuels, iconographiques, matériels). À propos des systèmes experts, il insista notamment sur le fait que ceux-ci ne concernaient pas exclusivement des objets textuels ${ }^{35}$. Rappelons aussi que sa chaire de direction d'étude à l'EpHe s'intitulait « Sémiologie et informatique » et, qu'à partir des années 1980, il s'est pleinement investit dans les débats accompagnant (également de manière critique) la diversification et le succès international de la sémiologie ${ }^{36}$.

Les deux auteurs admettaient ainsi, quoique pour des raisons différentes, que l'analyse des raisonnements ne peut se ramener à une sémiologie. Cela étant, ils s'opposaient à propos de la nature des termes employés dans les descriptions produites par les sciences de l'homme, soulevant les problèmes philosophiques relatifs aux concepts de description définie et de nom propre. Les deux auteurs se défendaient toutefois de se faire philosophes ${ }^{37}$ et se référaient à des philosophes de métier dont, en particulier, Granger. Ce sera donc avec Granger que nous commencerons pour une première clarification de ces concepts. Nous aborderons ensuite les conceptions respectives de Gardin et de Passeron à propos des termes employés dans les descriptions, puis les positions de ces deux auteurs sur le problème du degré de généralité des concepts admissible en sciences de l'homme.

privilégié.

34. Passeron 1991, p. 168.

35. Gardin 1987a, p. 239-241.

36. Depuis Saussure, la sémiologie désigne l'étude générale des systèmes de signes et de communication. Sur le renouveau de la sémiologie depuis les années 1980, $c f$. Gardin 2009, p. 175-176.

37. Plutniak 2017b, p. 12-13 


\subsection{Un problème philosophique implicite : descriptions définies et noms propres}

En 1989, Granger a consacré une étude aux fondements de la pensée symbolique. Il identifiait et discutait des conditions dites «proto-logiques » des langues naturelles, c'est-à-dire, des conditions non empiriques et nécessaires pour déterminer une fonction de symbole ${ }^{38}$. Parmi les cinq fonctions proto-logiques qu'il identifiait, figurait celle de nom propre, qu'il avait étudié dans un article antérieur. Dans ce dernier, il distinguait certaines spécificités des noms propres en les comparant aux descriptions définies, un type de description permettant de désigner, sans le nommer, un unique objet à partir d'un ensemble fini de traits descriptifs ${ }^{39}$. Granger remarquait que les éléments d'un système formel ne possèdent pas d'individualité au-delà de l'ensemble des traits pertinents retenus pour les décrire, c'est-à-dire au-delà de leur description définie. Il posait qu'il s'agit là de «pseudo-individus » (ou «types »), que l'on désigne par des pseudo-noms propres. Au contraire,

" [l]e nom propre, au sens strict, est un type de signe qui n'apparaît qu'en langue naturelle. Son remplacement par des descriptions, qui revient à en supprimer radicalement les effets originaux dans un système symbolique, caractérise l'entreprise scientifique d'approximation indéfiniment différée de l'individuel. ${ }^{40}$

Granger contrastait les noms propres de lieux qui peuvent, dans une certaine mesure, encore être substitués par une description (un système de coordonnées spatiales, un assemblage de noms communs), et le cas «pur» des noms propres de personnes, dont la propriété fondamentale est pragmatique : permettre l'interpellation d'une personne. Parmi les sciences, seules celles de l'homme sont confrontés à ce type de noms propres. Il en découle l'impossibilité d'employer des définitions définies en dernière instance dans ces sciences; ainsi que " [l]e nom propre ne saurait être complètement déterminé ni par des traits syntaxiques, ni par des traits sémantiques, mais par un trait pragmatique ${ }^{41}$. Granger étendait aux noms propres en général sa remarque concernant l'interpellation spécifique aux noms propres de personnes. Il identifiait pour cela une autre fonction protologique, celle d'ancrage. L'ancrage concerne «la trace, matérielle ou virtuelle, de l'auteur de l'énonciation en tant que tel dans l'énoncé, dans la mesure où cette trace en conditionne le sens. » ${ }^{42}$. L'ancrage se réalise par des « indicateurs de subjectivité », propres au langage naturel (les langues telles que le français, l'espagnol), qui font que « je est lié à l'énonciation de l'énoncé » ${ }^{43}$. Venons-en à la manière dont ces concepts sont employés dans les travaux et positions de Gardin et de Passeron.

\subsection{L'usage des descriptions définies en sciences de l'homme}

Le développement de l'analyse documentaire mené par Gardin dans les années 1950 et 1960 était une réponse aux problèmes posés par la diversité des concepts

38. Granger 1989.

39. Leur première caractérisation se trouve dans : Russell 1905.

40. Granger 1982a, p. 36.

41. Granger 1982a, p. 36.

42. Granger 1982a, p. 24.

43. Granger 1989, p. 253. 
descriptifs produits par les archéologues à propos de mêmes catégories d'objets. En outre, la masse grandissante de matériaux archéologiques décrits dans la littérature entraînait une dépense que Gardin jugeait excessive de temps pour les recherches documentaires. Elle pourrait, selon lui, être évitée par l'automatisation ${ }^{44}$. Entre 1956 et 1969, Gardin a supervisé la réalisation collective d'une quinzaine de «codes » de description, adaptés à l'analyse d'objets variés tels que la glyptique orientale, les mosaïques romaines ou les manuscrits romans. En 1965, il visait ainsi :

« [...] une autre forme de désignation, plus analytique, où l'on se bornerait à énoncer les éléments constitutifs de chaque monument, considéré sous différents points de vue - morphologique, fonctionnel, etc. - sans tenter de donner un nom à telle ou telle combinaison empirique de ces éléments. ${ }^{45}$

Cette définition correspond à celle des descriptions définies, et cet effort de stabilisation sémantique se retrouve également dans ses travaux ultérieurs : les systèmes experts qu'il développa dans les années 1970 et 1980 reposaient sur la définition standardisée d'une «base de faits » et d'une «base de règles »; la méthode de réécriture «logiciste », développée dans les décennies 1990 et 2000, consistait à reprendre les argumentations contenues dans des textes de sciences humaines en langue naturelle, pour les représenter par des arbres composés de propositions explicites et de relations d'inférences. Les principes de parcimonie et d'explicitation, propres aux descriptions définies, furent des constantes des travaux de Gardin.

\subsection{Les (semi-)noms propres des sciences historiques}

C'est contre la possibilité d'une telle stabilisation du sens des concepts descriptifs en sciences de l'homme que s'est érigé Passeron. Il chercha à établir une position médiane en développant une critique dirigée contre deux cibles dont, premièrement, les formes les plus radicales de réductionnisme linguistique. Il visait notamment les positions défendues par les tenants du linguistic turn à qui Passeron reprochait d'assimiler l'histoire à une science abstraite des symboles (une «tropologie $\gg)^{46}$. Il s'opposait deuxièmement aux ambitions de réduction logique du langage naturel, préalables à une représentation formalisée des raisonnements. Contre ces deux cibles, les travaux de Granger tenaient une place particulièrement importante dans les arguments développés par Passeron.

Parmi les cinq fonctions proto-logiques discutées par Granger, Passeron repris celle d'ancrage et celle de nom propre. Elles permirent notamment de fonder la thèse selon laquelle « [i]l n'existe pas et il ne peut exister de langage protocolaire unifié de la description empirique du monde historique $\gg{ }^{47}$. À partir de la conception du nom propre de Granger et de l'idée de sa détermination fondamentale sur le plan pragmatique, Passeron forgea la notion de semi-nom propre. Comme les noms

44. Plutniak 2017a, p. 119-120.

45. Gardin 1965, cité dans la réimpression Gardin 1974a, p. 83.

46. Passeron et al. 1996, p. 299-300.

47. Proposition 2, Passeron 1991, p. 363. La protocolarisation d'un langage correspond à la précision de la définition de ses termes et de ses règles, dont le degré le plus poussé serait la formalisation (Passeron 2006, p. 547). 
propres, ces semi-noms propres fonctionnent comme des « désignateurs rigides » au sens où ils permettent de désigner univoquement un objet, indépendamment de ses propriétés et de la façon de le décrire ${ }^{48}$. La caractérisation de ces semi-noms propres par Passeron présente toutefois certaines variations entre ses différentes publications. Il a pu ainsi les opposer aux descriptions définies en raison de cette dépendance à une action de désignation (il parle alors de leur caractère déictique) ${ }^{49}$; ou, ailleurs, indiquer que les semi-noms propres constituent des "mixtes logiques de "désignation" et de "description définie" " ${ }^{50}$, comme le seraient par exemple les concepts de féodalité ou de fascisme, comportant à la fois des propriétés génériques transhistoriques et de nécessaires indexations spatiotemporelles.

Toujours est-il que, selon Passeron, les caractéristiques des semi-noms propres induisent et fondent la spécificité du raisonnement historique (au même titre que les pseudo-noms propres de Granger déterminent les systèmes formels) ${ }^{51}$.

Plus généralement, l'impossibilité, selon Passeron, de faire référence aux choses du monde socio-historique à l'aide de descriptions définies, de définitions préalables, ou de définitions génétiques (qui permettent d'engendrer l'objet défini par des opérations logiques), détermine la spécificité des raisonnements menés en sciences historiques. Ces sciences ne disposent pas des conditions qui permettent, en d'autres sciences, de recourir à ces types de définitions, à savoir l'universalité formelle des opérations d'un système logique, et la généralité expérimentale ${ }^{52}$. Ce sont les concepts historiques, compte-tenu de leur nécessaire indexicalité aux choses du monde, qui permettent néanmoins de maintenir le projet d'une étude scientifique des réalités socio-historiques. Il n'en demeure pas moins que, pour ce qui concerne les raisonnements, la position de Passeron s'opposait à celle de Gardin : selon ce dernier, les propriétés des termes et des descriptions ne modifient pas la nature des raisonnements réalisés en sciences de l'homme.

\subsection{Le degré de généralité des concepts employés et ses conséquences sur les raisonnements}

Passeron posait que le « raisonnement naturel» procède par un «affinement inlassable des analogies » : c'est-à-dire que les critères d'équivalence permettant de comparer les choses entre elles sont sans cesse précisés par l'étude de nouveaux cas et par l'introduction de nouvelles distinctions analytiques ${ }^{53}$. Ce processus d'affinement a pour effet de réduire la généralité des théories fondées sur les observations empiriques. Toutefois, selon Passeron, cet effet est contrebalancé par

48. La notion de désignateur rigide a été proposée par Saul Kripke et discutée par Granger dans son article de 1982. Passeron reproduit simplement les références bibliographiques aux travaux de Kripke et de Keith Donnellan données par Granger.

49. Passeron 1991, p. 364 ; Passeron 2001b, p. 41.

50. Passeron 2002, p. 155 .

51. La reprise par Passeron de cette théorie du nom propre a pu être qualifiée de «prolongement ingénieux 》 : Lacour 2005, note 61.

52. Passeron 1991, p. 162 ; Passeron 2006, p. 268.

53. La même idée se rencontre chez Granger : «La réussite de la connaissance scientifique exige, croyons-nous, qu'on se résigne à cette réduction, et son progrès ne peut consister que dans l'enrichissement des référentiels et l'affinement du repérage. »(Granger 1982a, p. 27.) Voir aussi la citation du même article donnée précédemment, p. . 
la réalisation d' 'écarts sémantiques ${ }^{54}$ : pour admettre davantage d'éléments à comparer, l'analyste adopte des critères plus généraux permettant d'identifier un phénomène empirique observé à un type. Pour Passeron, l'ampleur donnée aux écarts sémantiques a pour effet de modifier la nature du raisonnement.

Dans les années 1990, Gardin émaillait ses textes de références à une idée de Bertrand Russell, celle de «crude uniformities » qui s'observeraient aux stades initiaux d'une investigation scientifique :

«En résumé, chaque progrès dans la science nous entraîne plus loin des uniformités grossières qui sont d'abord observées, dans une plus grande différenciation de l'antécédent et du conséquent, et dans un cercle continuellement élargi d'antécédents qu'on reconnaît comme pertinents. » ${ }^{55}$

L'affinement progressif admis par Passeron n'apparaît pas si différent du processus décrit ici par Russell. Contre Passeron, Gardin objecte néanmoins que le raisonnement et le degré de généralité de la description des objets sont deux choses distinctes. Certes, le niveau de généralité requis dans la définition des types - et des critères sur la base desquels associer un type et une occurrence empirique peut varier selon le type de phénomènes observés (matériel, mental, social, etc.). Il n'empêche que, selon lui, cela ne change rien aux mécanismes du raisonnement, et que ceux-ci sont les mêmes en sciences naturelles et en sciences de l'homme. Remarquons que Gardin n'approfondit pas particulièrement les spécificités qui seraient liées à la nature des phénomènes étudiés. La divergence des deux auteurs sur la question (épistémologique) du raisonnement s'accompagne d'une commune minoration des aspects ontologiques ${ }^{56}$.

$\stackrel{* *}{* *}$

L'examen de leurs conceptions respectives des propriétés des termes employés en sciences de l'homme nous conduit ainsi à un point d'opposition fondamental de leurs épistémologies : le problème de la spécificité des raisonnements menés dans ces sciences.

\section{La spécificité des raisonnements en sciences de l'homme}

La caractérisation d'une éventuelle spécificité épistémologique des sciences de l'homme a été l'enjeu principal des thèses de Passeron, l'accentuation du déterminisme historique constituant son principal moyen de démarcation par rapport aux travaux structuralistes. C'est aussi un aspect fondamental de la dispute alimentée avec Gardin, que nous aborderons en examinant trois points. Le premier concerne le rapport entre logique et faits historiques : la nature des phénomènes humains réclame-t-elle de recourir à une logique d'un genre particulier, laquelle impliquerait un mode de raisonnement spécifique? Passeron répondait par l'affirmative, Gardin par la négative; l'un et l'autre se référaient toutefois à la logique naturelle de Grize, que nous aborderons en deuxième point. Enfin, nous montrerons troisièmement que leur différend découle d'une divergence quant à l'unité d'analyse

54. Passeron 1991, p. 369-370.

55. Russell 1917, p. 188. Gardin renvoie de manière erronée à la p. 118, par ex. dans : Gardin 1993, p. 162. La version française ici citée est : Russell 2006.

56. Plutniak 2017b, p. 14-15. 
adoptée pour l'examen des raisonnements.

\subsection{Quelle logique pour raisonner sur les phénomènes humains?}

Dans son Raisonnement sociologique de 1991, Passeron rappelait la persistance, dans l'histoire de la pensée, d'une fascination pour le développement d'une langue universelle. Dans cette généalogie, il incluait différents avatars, depuis l'Ars Magna de Ramon Llull, la Caractéristique universelle de Gottfried Leibniz, jusqu'aux ambitions ayant accompagné le développement des ordinateurs. Passeron inscrivait enfin les travaux de Gardin dans cette filiation, considérant que "Dans sa forme extrême, ce projet ne vise à rien de moins qu'à une refonte complète de l'énonciation à partir d'un "modèle logique de langage scientifique". » ${ }^{57}$.

Examinons maintenant l'objet de cette critique, réitérée dans l'édition de 2006, en remarquant tout d'abord qu'elle est similaire à une critique adressée par Granger à Gardin dans une correspondance privée en 1973. Gardin lui avait communiqué son article « Document Analysis and Linguistic Theory», qui fit dire à Granger :

«Un seul point me paraît sujet à caution : c'est votre approbation de la "réintroduction de la logique symbolique comme formalisme convenable pour l'expression" des structures profondes. Bien entendu, on pourra toujours traduire plus ou moins des schémas considérés comme constituant une structure profonde au moyen de formes logiques. Fe crois cependant qu'il s'agit là d'un lit de Procuste. Les formalismes logiques connus me paraissent être issus d'une analyse de ce que vous appelez le discours, dont ils constituent l'un des niveaux d'organisation. Quant aux structures profondes, si l'on veut continuer à faire usage du mot logique à leur égard, ce ne peut être qu'en un sens différent. (C'est un peu ce que fait Wittgenstein lorsque, dans le Tractacus, il parle de la logique des propositions élémentaires, par opposition à la logique - au sens usuel des assemblages de propositions). Bref, ma conjecture est que l'analyse de ces structures à la fois proto-sémantiques et proto-syntaxiques (il me semble que nous serions d'accord sur ces dénominations?) doit conduire à des formalismes nouveaux, irréductibles par exemple aux "prédicats" et "relations" classiques; d'où les difficultés. »" 58

On remarquera au passage que Gardin parle en fait de formalisme « commode » (convenient dans la version originale en anglais) et non de formalisme " convenable ${ }^{59}$. La nuance est de taille car elle fait basculer de l'idée d'adéquation (ontologique) à celle d'efficacité (pratique et épistémologique). Nonobstant cette nuance, la perspicacité de la critique similaire formulée en 1991 par Passeron était donc toute relative : elle touchait un point que Gardin était peu susceptible d'ignorer, puisque déjà signalé en 1973 par Granger. En outre, entre-temps, Gardin avait largement nuancé sa position, au fil des différents programmes de recherche qu'il a animés. Ses travaux sur les langages et systèmes documentaires, puis ceux sur les systèmes experts et, enfin, son programme logiciste se sont, certes, inscrits dans une certaine continuité. Ils se sont toutefois également fondés sur les difficultés

57. Passeron 1991, p. 154 .

58. Lettre de G.-G. Granger à J.-C. Gardin, 07-11-1973, MAE.

59. Voir respectivement Gardin 1973, p. 158; Gardin 1974b, p. 164. 
rencontrées et les réorientations effectuées en conséquence ${ }^{60}$. Dans un article de 1971, Gardin exprimait une conception qui resta commune à ses différents travaux :

«Cet effort d'analyse et d'objectivation n'a pas pour but, on s'en doute, de mettre en évidence les failles de la pensée d'autrui... Il trouve aussi bien à s'exercer dans la voie d'une introspection systématique, par où chacun tenterait de formuler aussi clairement que possible les données sur lesquelles il opère, la visée de la recherche, les hypothèses sous-jacentes au raisonnement, les mécanismes logiques utilisés, etc. [...]. L’archéologie ne fait alors que retrouver le chemin de la science en général. Qu'il y soit conduit ou ramené par l'informatique n'est pas un mal en soi; mais les nouvelles perspectives qui s'ouvrent à lui sont d'abord celles d'une pensée bien conduite [...] avant d'être celles de l'électronique. On s'en convaincra sans peine, s'il est besoin, en considérant d'une manière également critique les résultats obtenus par la machine, lorsque machine il $y a:$ il reste en effet à prouver que ceux-ci ont un sens quelconque, du point de vue de la connaissance archéologique, le caractère objectif de la démarche suivie pour les acquérir n'étant aucunement une garantie de leur utilité scientifique. ${ }^{61}$

En 1987, dans un ouvrage collectif à propos des systèmes experts, Gardin récapitula lui-même six objections - qu'il jugeait valides - contre le recours à cet instrument. Les critiques formulées par Passeron en 1991 recouvraient en partie ces objections. Gardin soulignait qu'il serait malgré tout erroné de condamner entièrement les systèmes experts dans la mesure où les limites pointées dans ces six points

«[...] ne font que traduire les propriétés ou impropriétés, selon les cas, des modes de construction symbolique propres à la science en général et aux sciences humaines en particulier. ${ }^{62}$

Ces limites seraient alors inhérentes au raisonnement en général, et ne constituent donc pas un argument en faveur de la thèse d'une forme de raisonnement spécifique aux sciences humaines. Les nuances et précautions de cette sorte, répétées par Gardin dans ses diverses publications, n’ont pas particulièrement été prises en compte par Passeron. Dans ses critiques, ce dernier signalait l'ouvrage collectif sur les systèmes experts. Toutefois, il ne s'appuyait pas sur les chapitres introductifs signés par Gardin, dans lesquels celui-ci précisait ses options épistémologiques et méthodologiques. Passeron se limitait, au contraire, à citer les brèves remarques contenues en introduction de l'étude de cas rédigée par Gardin dans ce même volume.

Les difficultés rencontrées dans les expérimentations avec les systèmes experts ont conduit Gardin à approfondir par la suite son programme dit « logiciste ». Ce programme comprenait une méthode de réécriture des argumentations scientifiques en langage naturel sous la forme d'arbres constitués de propositions liées par des relations d'inférence. Gardin a insisté à de nombreuses reprises sur le fait

\footnotetext{
6o. Gardin 1988.

61. Gardin 1971, p. 215.

62. Gardin 1987a, p. 238.
} 
que ces "opérations de dérivation sont étrangères à la logique formelle et aux mathématiques » et, partant, sur leur différence avec les inférences automatiques réalisées par un système expert ${ }^{63}$. Ou encore que :

"L'analyse logiciste n'est pas une tentative de refonte du discours savant dans les moules de telle ou telle logique formelle. Elle repère et respecte les enchaînements que pratiquent les auteurs des textes visés, dans la syntaxe qui est la leur. La schématisation vise seulement à isoler les opérations d'inférence ou de réécriture auxquelles se ramènent ces enchaînements, débarrassés de leur expression rhétorique. ${ }^{64}$

Or, Passeron considérait indistinctement les deux projets et négligeait la particularité de l'approche logiciste ${ }^{65}$. Un sociologue a, lui aussi, ultérieurement souligné la manière simplificatrice dont Passeron résumait les travaux menés en intelligence artificielle ${ }^{66}$. Ce dernier réitérait donc en 1991 la critique exprimée par Granger en 1973, mais sans tenir compte de l'évolution des travaux menés par Gardin entretemps. La mise en scène, par Passeron, d'une franche opposition entre leurs théories résiste ainsi difficilement à une lecture scrupuleuse, bien que l'on comprenne qu'elle permette une démarcation commode avec des travaux supposément incompatibles faisant fonction de repoussoir.

En somme, Passeron considérait qu'en sciences humaines l'indexicalité des concepts et la dépendance des raisonnements à leur contexte d'énonciation donnait lieu à un type de raisonnement particulier, dit «naturel». Ce raisonnement « naturel » revient-il au raisonnement en langage naturel? Selon Gardin, Passeron laissait ce point sans réponse, d'où les embarras du premier. Afin d'éclaircir cet aspect, un détour par les recherches de Grize sur la logique naturelle, auxquels les deux auteurs se référaient, est donc nécessaire.

\subsection{Les usages d'une référence commune : la logique naturelle}

Dans son effort de caractérisation du raisonnement historique, Passeron posait que :

«Par ses schèmes d'inférence comme par ses concepts descriptifs, le raisonnement sociologique fonctionne comme un "raisonnement naturel", c'est-à-dire comme un raisonnement dont la sémantique ne peut jamais être entièrement coupée de celle d'un "langage naturel". » ${ }^{67}$

Il faisait ensuite immédiatement référence à Grize dans une note, créditant ce dernier d'avoir

«[...] montré que les mouvements sémantiques les plus caractéristiques

d'une "logique naturelle" étaient irréductibles aux opérations d'une logique pure des classes, des prédicats ou des propositions. »

De cette manière, Passeron distinguait clairement le raisonnement historique de celui pratiqué en logique. Au détour d'une autre référence aux travaux de Grize, il

\footnotetext{
63. Gardin 1991a, p. 71.

64. Gardin 1997a, p. 59-6o.

65. Passeron 1991, p. 373.

66. Chateauraynaud 2003, p. 171-172.

67. Passeron 2006, p. 59.
} 
précisait davantage la teneur de sa reprise de la logique naturelle. Il indiquait alors que l'association spatio-temporelle indéfectible entre une description historique et l'objet du monde visé constituait une opération de «logique naturelle ${ }^{68}$. Voyons maintenant les reprises des travaux de Grize par Gardin.

Le concept de logique naturelle a été travaillé, avant Grize, par d'autres auteurs, dont le linguiste George Lakoff (1941-). En référence à ce dernier, Gardin exprimait déjà en 1974 son embarras face à l'idée selon laquelle il existerait une logique dont les propriétés justifieraient de la distinguer et de la qualifier de «naturelle ». Gardin concluait qu'une telle logique pouvait être, in fine, ramenée à la logique standard : "Bien que le contenu de cette "logique naturelle" ne soit pas des plus clairs, on peut admettre que le calcul des prédicats et le calcul des propositions de la logique standard en constituent des approximations convenables $\gg{ }^{69}$. Au cours des années 1980 et 1990, Gardin a entretenu des échanges intellectuels réguliers avec Grize. Il a participé à un volume collectif, dirigé par ce dernier, sur l'intelligence artificielle et l'analyse de discours ${ }^{70}$, a aussi contribué à deux volumes en son hommage, offrant dans chacun une discussion critique de la logique naturelle ${ }^{71}$.

En dépit de ses réticences quant à la logique naturelle, mais grâce au dialogue qu'il entretint avec Grize, Gardin a réalisé plusieurs emprunts conceptuels aux travaux de ce dernier. La logique naturelle de Grize comportait une méthode de réduction et de représentation des raisonnements sous la forme de «schématisations ». Gardin y fit référence et adopta le même terme pour désigner la forme schématique de réécriture des raisonnements préconisée dans sa méthode logiciste ${ }^{72}$. Il reprit également l'idée de « procédé d'étayage » que Grize proposait pour désigner l'implication par laquelle un énoncé $Q$ est obtenu par un « raisonnement » opéré sur un énoncé $P$ (sous la forme si $P$ alors $Q$ ) ${ }^{73}$. La réécriture logiciste consistait à établir de telles relations entre les propositions de plus en plus générales extraites d'un texte scientifique en langue naturelle.

Passeron et Gardin ont donc retenu des aspects différents de la logique naturelle de Grize. Examinons l'avis de ce dernier quant à ces recours. Grize se proposait d'examiner l'équivalence entre la logique naturelle et le raisonnement «sociologique » (ou «naturel ») de Passeron. Il concluait, confortant les thèses de ce dernier, que la logique naturelle contribuait à distinguer le discours sociologique de celui des sciences physiques et naturelles ${ }^{74}$.

Grize se montrait par contre moins favorable par rapport à la reprise de la logique naturelle réalisée par Gardin. Il reconnaissait, certes, que le logicisme de Gardin et sa logique naturelle avaient en commun, comme toute axiomatique, de porter sur des « organisations logico-sémantiques », c'est-à-dire des « articulations contrôlées des contenus ${ }^{75}$. Toutefois, dans un article spécifiquement dédié aux travaux de Gardin, Grize pointait une différence fondamentale relative à la nature des éléments auxquels est appliqué cette axiomatique : «Un contenu de jugement n'est

\footnotetext{
68. Passeron 2001b, p. 40 .

69. Gardin 1974b, p. 164 .

70. Gardin 1987b.

71. Gardin 1987c; Gardin 1997a.

72. Gardin 2001, p. 459.

73. Gardin 1997a, p. 44. Gardin cite Grize 1995.

74. Grize 1994b, p. 286-289.

75. Grize 1996, p. 81-82.
} 
ni vrai ni faux en lui-même et en conséquence ne fournit pas un fait. Celui-ci ne peut résulter que de son assertion par un locuteur. ${ }^{76}$. Or, «[d]ans les nouds des schémas de Jean-Claude Gardin figurent des classes-objets ou des contenus de jugement, jamais des énoncés ». Ceci constituait une différence fondamentale avec l'approche développée par Grize, laquelle concernait non pas des propositions mais des énoncés. Passeron s'opposait également à Gardin sur le même point.

\subsection{Propositions et énoncés : divergences sur l'analyse des raisonne- ments}

Passeron accordait un statut particulier à la distinction entre raisonnement formel et raisonnement «naturel », distinction que Gardin récusait ${ }^{77}$. Pour reprendre le commentaire de Gérard Lenclud, le premier de ces raisonnements «s'impose au titre de la nécessité logique », se livre par un modèle logico-mathématique et développe une démonstration; le second «cherche à persuader au nom de la plausibilité », se livre par une argumentation qui conduit à une preuve ${ }^{78}$.

Ce point d'opposition entre les deux auteurs à propos des rapports entre les niveaux logique, épistémologique, et linguistique est fondamental. Là où Passeron abordait les raisonnements en distinguant les assertions (des propositions ayant une valeur de vérité) et les énonciations (des actes langagiers situés), Gardin considérait indifféremment des propositions (similaires aux assertions de Passeron). Il s'agissait donc de parti pris opposés concernant l'unité d'analyse privilégiée pour l'étude des raisonnements.

Gardin admettait un certain degré d'autonomie entre les dimensions cognitives, linguistiques et historiques ${ }^{79}$. Poussé par les réactions hostiles à ses propositions, il a émaillé ses textes de clarifications concernant les déterminations des raisonnements par des facteurs externes. Dans le livre collectif sur les systèmes experts, il affirmait que l'analyse des formes discursives et l'analyse des raisonnements sont deux objets différents, et que ses travaux ne concernaient que la dernière. Cette distinction n'interdit en rien que d'autres investigations s'attachent à des facteurs externes au raisonnement, qu'ils soient linguistiques, psychologiques, ou sociaux ${ }^{80}$. Dans un texte ultérieur, il posait que les ouvres et conduites humaines (e.g. un mariage observé), les catégories permettant leur analyse (le concept d'alliance), et les vues de l'autre sur ces œuvres et conduites (la théorie émique du mariage considéré) constituent trois catégories d'objets, susceptibles d'investigations scientifiques distinctes ${ }^{81}$.

En conséquence, Gardin assumait une indistinction entre assertion et énonciation, privilégiant le contenu d'une proposition plutôt que ses modalités pragmatiques de réalisation. Ce préalable conceptuel permettait de distinguer les objets et les arguments à leurs propos et facilitait la modélisation de leurs rapports. Dans le domaine des systèmes experts, auquel se consacra Gardin dans les années 1980,

76. Grize 2000, p. 133 .

77. Gardin 1993.

78. Lenclud 2001, p. 451.

79. Gardin 1987a, p. 240.

8o. Gardin 1987 c, p. 195, en particulier la note 42.

81. Gardin 1991b, p. 101. L'exemple du mariage est de mon fait. 
ceci se traduisait par la conception de deux bases de données, l'une pour les faits, représentant les objets, et l'autre pour les règles d'inférence, correspondant aux raisonnements ${ }^{82}$. Le système expert reproduisait ainsi le fonctionnement cognitif d'un expert grâce à un moteur d'inférence permettant d'appliquer les règles aux faits et de générer de nouveaux faits. Admettre la distinction entre objet et raisonnement constituait un préalable à l'utilisation du calcul afin de désambiguïser les interprétations polysémiques dans les raisonnements des sciences de l'homme et, in fine, être en mesure de décider entre des théories concurrentes. Cette distinction n'implique toutefois pas - Gardin s'en gardait bien - de considérer comme possible une science générale des raisonnements fondée sur la logique ou sur la sémiotique ${ }^{83}$.

Inversement, selon Passeron, les assertions en sciences historiques sont nécessairement des énoncés. Il considérait le langage comme un

$$
\begin{aligned}
& \text { "[...] moyen d'exprimer et de transmettre des significations articulées } \\
& \text { entre elles en fonction de la situation de parole et du déroulement du } \\
& \text { message, c'est-à-dire comme un acte d'énonciation. }{ }^{84}
\end{aligned}
$$

L'importance de ce point de divergence fut telle qu'il constitua la raison de la modification du sous-titre du Raisonnement sociologique pour la seconde édition, dans laquelle Passeron reconnaissait à Gardin un rôle décisif parmi les critiques de ces thèses ${ }^{85}$. Le raisonnement sociologique n'était plus l'espace non-poppérien nécessaire d'un type de raisonnement (L'espace non-poppérien du raisonnement naturel) mais celui contingent d'un genre énonciatif, l'argumentation (Un espace non-poppérien de l'argumentation). En passant du raisonnement à l'argumentation, Passeron renforçait le poids du caractère historique en tant que fondement de son épistémologie. Selon lui, le fait que les propositions des sciences historiques soient des énoncés entraîne qu'ils ne peuvent - même pour les plus simples - être formalisés complètement, leur sens étant redevable, même à minima, de la sémantique du langage naturel dans lequel ils sont exprimés :

«[...] il faudrait, pour définir les rapports entre une langue naturelle et une langue artificielle au sein d'un système formel, formaliser d'abord le fonctionnement de la langue naturelle par un système formel qui entretiendrait du même coup des rapports non formalisés avec la langue naturelle considérée. Ad infinitum. ${ }^{86}$.

Selon Passeron, les tentatives de formalisation menées par Gardin violaient directement cette limitation.

L'examen des conceptions du raisonnement en sciences de l'homme par nos deux auteurs permet donc d'identifier des points de divergence fondamentaux, concernant en particulier le statut accordé à la logique, et l'indexicalité - non seulement des descriptions - mais aussi des propositions (envisagées comme des énoncés). Le problème du degré de dépendance historique des propositions (à leurs

82. Gardin 2001, p. 459.

83. Gardin 1995, p. 28 et 30.

84. Passeron 2002, p. 171

85. Passeron 2006, p. 19.

86. Proposition 1.1, Passeron 1991, p. 361. 
contextes de référence et d'énonciation) est davantage qu'un problème philosophique. Il possède des implications pour la pratique des sciences de l'homme : la possibilité de recourir à la formalisation dépend en effet de la manière dont on considère et admet la désindexation des propositions.

\section{Le juste degré de formalisation du langage et des raison- nements}

Les concepts de «formalisation », « modélisation », «protocolarisation », « standardisation » ou « axiomatisation »- tous présents dans la controverse entre Passeron et Gardin - ont fait l'objet de nombreux travaux de philosophie des sciences ${ }^{87}$. Toutefois, les rapports entre ces travaux et les discussions épistémologiques de praticiens des sciences de l'homme sont parfois ténus. La formalisation constitue pourtant une ligne de démarcation dans ces débats épistémologiques, qu'il s'agisse d'en pointer les errances (Passeron fustigeant les «Paradis artificiels du formalisme » dans le chapitre éponyme du Raisonnement sociologique) ou d'en défendre les vertus et le caractère indispensable (Gardin avançant le gain en consistance et en parcimonie ainsi obtenu pour les raisonnements). Les enjeux de la dispute entre ces deux auteurs doivent donc être réinscrits dans un débat plus large tenu par plusieurs collectifs de chercheurs en sciences de l'homme au cours des années 1990. Tous deux ont notamment contribué à un séminaire intitulé « Le modèle et le récit ». Comme son nom l'indique, il a été conçu à partir de l'opposition entre ces deux modalités de la pratique des sciences de l'homme. L'un des mérites du livre collectif publié à l'issue de ce séminaire a été de rassembler des perspectives diverses sur l'antagonisme proposé au débat. La contribution d'un des organisateurs du séminaire, le sociologue Claude Grignon (1936-) - par ailleurs proche collaborateur de Passeron - abordait la formalisation dans une section consacrée $\mathrm{au}$ «passage à la formalisation : les mathématiques comme langage ». Comme ce titre l'indique, les trois concepts de formalisation, de mathématique et de langage étaient envisagés dans leurs rapports :

" [l]a formalisation, dont l'usage s'est imposé en économie, implique au contraire, du moins en principe, le primat du symbolisme logico-mathématique sur la langue naturelle. Plus un article est formalisé, plus celle-ci passe au second plan, plus la place (et le soin) qui lui est accordée tend à se réduire, au point que les formalisations les plus raffinées reposent parfois sur des intuitions confuses, non formulées, que l'on rejetterait sans doute si elles se présentaient sans déguisement, sous la forme explicite d'un récit. ${ }^{88}$

La spécificité de cet argument peut être contestée en intervertissant les termes, afin de faire valoir que : les récits les plus raffinés reposent parfois sur des intuitions confuses, masquées par des nuances de la langue, que l'on rejetterait sans doute si elles se présentaient sans déguisement, sous la forme explicite d'une expression formalisée. On conclura que si l'argument vaut dans les deux cas, c'est

87. Pour une brève clarification de ces concepts, voir Mongin 2003, p. 106-110.

88. Mes italiques. Grignon 2001, p. 10-11. 
que le problème ne se pose finalement pas en termes d'une alternative entre récit et formalisation ${ }^{89}$. Tout du moins, le passage cité illustre une ambiguité fréquemment entretenue à propos de la notion de formalisation. On y retrouve à la fois l'idée qu'elle correspond à un changement de nature du raisonnement (supposant un "passage », comme l'indique le titre ${ }^{90}$ ), et l'idée qu'elle est susceptible d'une gradation (comme dans le passage cité). La dispute de Passeron et Gardin a porté sur ces problèmes, que nous aborderons d'abord à propos de la formalisation des descriptions, puis de celle des raisonnements et des modèles. Enfin, nous montrerons que l'opposition entre ces deux auteurs et, par extension, entre ces deux conceptions des sciences de l'homme, est moins franche que le prétendait Passeron.

\subsection{La formalisation des descriptions}

L'embarras principal de Gardin face aux propositions de Passeron tenait à la contradiction qu'il relevait entre, d'une part, la revendication d'une « langue théorique de description » et, d'autre part, l'affirmation de l'impossibilité de tout langage « protocolaire » en sciences de l'homme; thèse à laquelle Passeron ajoutait un jugement affirmant le caractère de toute manière indésirable de ces langages ${ }^{91}$. Qu'elles soient dites «théoriques » ou « protocolaires » ces langues étaient considérées équivalentes par Gardin et, dans tous les cas, en rupture avec le langage et le raisonnement dits «naturels » :

«[...] j'ai pour ma part de la difficulté à concilier l'apologie d'“une langue théorique de description" ou d'une "grille conceptuelle de description" pour l'expression de nos constats empiriques avec les nombreuses propositions antérieures touchant le caractère inaccessible et au demeurant indésirable de tout langage "protocolaire" dans les sciences de l'homme. » ${ }^{92}$

Gardin pointait la difficulté à distinguer l'explicitation propre à une «langue théorique de description» que peut se donner, selon Passeron, le sociologue ou l'historien, et l'explicitation que suppose la définition d'un système de description tel qu'un code, un système expert, une simulation par automates cellulaires ou par agents. Selon cette approche, la «formalisation » peut porter sur la formalisation que constitue déjà toute langue naturelle, dans la mesure où une langue comporte nécessairement l'articulation de structures à différents niveaux (phonologique, syntaxique, sémantique) ${ }^{93}$. Plus généralement, la mathématisation de méthodes peut concerner les mathématiques elles-mêmes : étant donné la diversité des mathématiques, cela revient à l'application d'une de ses parties à une autre ${ }^{94}$. Chateauraynaud, discutant les thèses de Passeron, écrivait également : «Et au fond la langue artificielle dont parle Passeron n'est-elle pas une possibilité contenue dans

\footnotetext{
89. Voir l'argument développé à ce propos dans une contribution critique au séminaire : Stoczkowski 2001.

90. Voir aussi Grignon 2001, p. 12.

91. Gardin se réfère à la proposition 3·3.1.1 des Propositions et scolies : Gardin 1993, p. 163.

92. Gardin 1993, p. 163.

93. La langue naturelle permet de construire des modèles qualitatifs dont le caractère scientifique n'est, selon Granger, pas moindre à celui des modèles quantitatifs : Granger 1982b.

94. Roux 2010, p. 336. Voir également l'idée de remathématisation avancée pour caractériser certains usages de la simulation informatique (Varenne 2008).
} 
la langue naturelle elle-même? ${ }^{95}$. Dans la perspective de Gardin, le problème n'est pas tant de distinguer langage naturel et langage artificiel mais, plutôt, de pratiquer le degré de formalisation le plus adéquat aux besoins des sciences de l'homme.

Ayant rendu sa construction théorique dépendante d'une distinction franche entre langage naturel et langage artificiel, Passeron se trouvait contraint d'adopter des positions radicales et, parfois, frustes à propos de la formalisation. En 1993, lorsque Gardin a envoyé à Granger sa note critique des thèses finales du Raisonnement sociologique, ce dernier répondit :

« Je vous remercie de l'envoi de votre article sur le livre de f.C. Passeron. fe me trouve en accord avec vous sur la plupart des points. Il me semble que cette très salutaire auto-critique des démarches et des ambitions de la sociologie va un peu trop loin dans son quasi-rejet du formalisme et son assimilation de la connaissance des faits humains à un jeu de langage "naturel". » ${ }^{96}$

Les positions générales de Granger à ce propos souffraient peu d'ambiguïté et se caractérisaient par leur constance dans le temps ${ }^{97}$. Dans les années 1960, il faisait débuter sa réflexion en reconnaissant que, d'une part, «il n'y a pas de science sans symbolisme articulé ${ }^{98}$ et que, pour ce qui concerne le rapport entre les dimensions orale et écrite du langage, "[l]'écriture, dans l'expression scientifique, n'est plus un code : elle est l'étoffe même du langage. », dans le sens où la structuration qu'elle permet est une condition nécessaire de la scientificité ${ }^{99}$. Quant aux rapports finaux entre langue formelle et langue naturelle dans l'exposé des propositions, il notait que le langage usuel ne peut «servir que de commentaire et d'accompagnement, commentaire du reste indispensable, dans la mesure où il permet de lier la syntaxe conventionnelle du symbolisme aux opérations et aux résultats expérimentaux. ${ }^{100}$. L'idée n'est pas fondamentalement différente de la thèse principale de Passeron concernant la formalisation, exprimée dans la selon laquelle « [l]a sociologie, comme l'histoire ou l'anthropologie, ne peut parler, dans ses énoncés finaux, qu'en langue naturelle. » ${ }^{101}$. La position de Granger semble seulement défaite du dramatisme et du souci insistant de distinction dont sont empreintes les formulations de Passeron. Granger reconnaissait sans peine l'insuffisance d'un langage formel seul et la nécessité d'un commentaire en langue naturelle; de plus, il donnait une raison à la fois sémantique et épistémologique à cette dépendance, le tout sans avoir à se défaire de l'idée saussurienne, "forte et simple, selon laquelle toute tentative pour connaître objectivement quelque chose de l'homme doit d'abord passer par une réduction de l'expérience à un système de marques corrélatives ${ }^{102}$. On peut reconnaître, derrière cette idée de réduction à un système de marques corrélatives

\footnotetext{
95. Chateauraynaud 2003, p. 172.

96. Lettre de G.-G. Granger à J.-C. Gardin, 12-07-1993, MAE.

97. Lacour 2005.

98. Granger 1967, p. 4 .

99. Granger s'opposait par cela à la restriction du statut de langage au seul langage oralisé, position alors défendue par le linguiste Leonard Bloomfield et ses adeptes : Granger 1967, p. 43.

100. Granger 1967, p. 50.

101. Proposition 2.4, Passeron 1991, p. 373.

102. Granger relevait cette idée alors qu'il discutait le concept de structure chez Saussure (Granger 1967, p. 43).
} 
(ou, autrement dit, une représentation par un ensemble de symboles liés par des relations structurées), une certaine acception du concept de modèle. Il reste à examiner maintenant la formalisation lorsqu'elle concerne non plus les descriptions, mais les raisonnements modélisés.

\subsection{Les modèles et la formalisation des raisonnements}

Dans sa contribution au séminaire Le modèle et le récit, Passeron proposa une typologie des modèles scientifiques. Il distinguait les modèles «purs » (ou « universels ») et les modèles à « déictiques » (ou à « désignation»). Les premiers comprennent des descriptions définies et sont exempts de tout nom propre, ils permettent de mener des expériences toutes choses égales par ailleurs, et peuvent être réfutés. Les seconds

\section{«[...] exigent, pour prendre un sens explicatif, que leur soient associées des descriptions faisant intervenir d'autres informations que celles sim- plifiées et sélectionnées pour les besoins de la modélisation. ${ }^{103}$}

Les distinctions employées dans la typologie des modèles de Passeron recouvrent ainsi celles employées pour sa typologie des concepts descriptifs.

Granger, soutenant qu'il ne pouvait y avoir de science que du structurable, a pu simplement définir les modèles comme des représentations abstraites et structurée des phénomènes ${ }^{104}$. En outre, selon lui, modéliser n'implique pas nécessairement de quantifier, et la portée d'un modèle n'est pas déterminée par le fait d'être quantitatif ou qualitatif. Cette question de la portée (ou de la généralité) constituait justement une différence importante entre les conceptions de la modélisation respectivement défendues par Passeron et Gardin. Ce dernier voyait dans la portée un critère décisif pour établir la possibilité des modèles en sciences de l'homme.

Gardin écartait d'emblée toute idée d'un possible modèle général ou universel. Lui attribuer une telle ambition, comme le faisait Passeron, relève plus de la caricature que de l'analyse épistémologique. Loin de souscrire à pareille ambition, Gardin, dans son article - déjà signalé - discutant de la logique naturelle de Grize, se prononçait en faveur des « field-related logics » proposées par le philosophe des sciences Stephen Toulmin (1922-2009) ${ }^{105}$. Ces «logiques de champs » (selon sa traduction) désignent des ensembles d'opérations logiques dont la portée est limitée à un domaine empirique particulier mais qui sont néanmoins distinctes du « raisonnement naturel » propre au discours ordinaire ${ }^{106}$. Même un philosophe rationaliste tel que Granger, fermement attaché à la possibilité d'une science de l'homme, écrivait en 1969 - période autrement plus propice à une conception scientifique des sciences de l'homme - que ces sciences

« [...] s'efforcent bien de déterminer, à différents niveaux hiérarchisés ou enchevêtrés, des structures, définies en tant que telles comme systèmes logiques. Mais la thèse ici présentée pose que nulle objectivation scienti-

\footnotetext{
103. Passeron 2001a, p. 245.

104. Granger 1982b.

105. Gardin 1987 c, p. 195.

106. Gardin se réfère à la conclusion de Toulmin 1958. Voir aussi, contre Passeron : Gardin 1997b.
} 
fique n'est possible pour la totalité de l'expérience et que nulle superlo-

gique ne gouverne l'ensemble de ces structures. ${ }^{107}$

En somme, Passeron et Gardin s'opposaient en faisant valoir, l'un, des types distincts de modèles, l'autre des modèles divers mais satisfaisant à des principes communs. J'insisterai maintenant sur les arguments qui, dans leur dispute, conduisent pourtant à nuancer leur opposition.

\subsection{Des formalisations qui s'ignorent}

Les deux auteurs, dans leurs accusations réciproques d'excès - que cet excès concerne le dépouillement ou la surcharge sémantique des mots - en vinrent à outrepasser ce que leur rival a pu effectivement écrire. Ainsi, dans le détail, le refus de la formalisation de Passeron prit des tours moins tranchés. « À condition de le savoir », écrivait-il, la formalisation, qualifiée premièrement de «contrôle illusoire », peut être employée même si

« [...] une langue artificielle à qui on confie un raisonnement sur la réalité historique risque toujours de faire oublier aux naïfs qu'elle recouvre, sous les apparences irréprochables de son symbolisme, autant d'ambiguïtés sémantiques. » ${ }^{108}$

Ainsi, Passeron n'établissait ou n'identifiait aucune nécessité logique entre l'emploi d'un langage formel en sciences historiques et les usages coupables qu'il indiquait. Tout au plus existe-t-il un risque, une probabilité; mais l'on reste sans savoir ce que la « naïveté » de ces réceptions et usages recouvre exactement. Ceci importe finalement peu, si l'on admet que la mise à l'index des usages « naïfs », « sauvages », « spontanés » ou «non-réflexifs » des concepts vaut avant tout pour anathème et moyen de démarcation commode des frontières du scientifique. Il est, de plus, intéressant de noter que la «naïveté » en question semble, si l'on suit Passeron, être déterminée par les langues ou les nations : en témoigne la traduction du titre du Raisonnement sociologique en espagnol, El razonamiento sociológico. El espacio comparativo de las pruebas históricas. La référence à Popper, sensée, selon Passeron, suggérer le « scientisme bête et ringard » au lecteur français fut gommée, au motif de ne pas posséder cette connotation pour le lecteur espagnol ${ }^{109}$.

Dans sa critique des moyens « illusoires » de contrôle en sciences historiques, Passeron reconnaissait explicitement que Gardin a pu formuler une critique similaire, en l'occurrence, à travers ce que ce dernier nommait «tests de pertinence » en analyse du discours ${ }^{110}$, gardes-fou à une délégation complète de l'interprétation au calcul automatique ${ }^{111}$. Le recours à la formalisation comme moyen de contrôle fut ainsi un objectif explicite des formalisations logicistes de Gardin : " [l]e recours aux machines serait, ici encore, un moyen de contraindre l'anthropologue à une connaissance plus explicite de ses propres démarches. ${ }^{112}$.

107. Granger contestait alors l'ambition universaliste de la logique dialectique. Granger 1969, p. 397 .

108. Passeron 1991, p. 156.

109. Moreno Pestaña 2011, p. 9.

110. Gardin 1974c, p. 16 et 39; Gardin 1974b, p. 139-140.

111. Passeron a maintenu cette analogie dans les trois versions de son texte: Passeron 1980, p. 140; Passeron 1991, p. 158; Passeron 2006, p. 263.

112. Gardin 1974a, p. 86 
Grize, dans une discussion du recours aux formalisations en sociologie menée à partir du Raisonnement sociologique de Passeron, adoptait une perspective similaire. Il pointait ainsi une fonction épistémologique plus fondamentale que les positions extrêmes consistant à considérer ces recours soit comme des moyens d'atteindre l'objectivité, soit comme des marqueurs rhétoriques de scientificité :

«C'est que les formalisations et les simulations informatiques - qui sont d'ailleurs fondamentalement de la même nature - constituent le moyen le plus sûr de prendre conscience des limites de ce que l'on fait et, en conséquence, de se mettre en état de les dépasser. Pour la théorie, sinon pour la pratique, les modèles logico-mathématiques importent bien davantage par ce dont ils ne rendent pas compte que par ce qu'ils saisissent. ${ }^{113}$

Quand bien même Passeron admettait certains recours à la formalisation, il insistait sur le fait que l'énonciation finale ne peut être réalisée qu'en langage naturel. $\mathrm{Ni}$ Gardin ni Granger ne récusaient ce point. La valeur probatoire du raisonnement historique devait alors, selon Passeron, reposer sur la sémantique de ces énoncés et, en particulier, sur les relations qui lient les termes dans les raisonnements énoncés par l'analyste. Toutefois, Gardin soulignait son embarras à ne pas voir Passeron préciser la nature de ces relations, l'amenant à considérer que leurs propositions respectives étaient finalement équivalentes.

Dans les Propositions et scolies du Raisonnement sociologique, Passeron suggérait un exercice de « commutation énonciative » visant à montrer que les effets des variations énonciatives sur la communication scientifique interdisent de réduire une énonciation à une structure logique unique :

« [...] changez, par une opération de déflation sémantique et de simplification du vocabulaire, les énoncés de surface, et observez à quel moment "commute" l'intelligibilité théorique de l'assertion et/ou l'information empirique qu'elle organise. ${ }^{114}$

Dès sa note critique de 1993, Gardin remarquait que cet exercice revenait tout simplement aux réécritures logicistes que lui-même promouvait ${ }^{115}$. Plutôt qu'une opposition théorique franche, il considérait ainsi une possible complémentarité ${ }^{116}$, une «superposition» des deux approches : celle de Passeron «ne s'écartant guère en principe des jeux de science pratiqués » dans l'approche logiciste ${ }^{117}$.

Ces considérations nuancent la mise en scène par Passeron d'une irréconciliable différence entre son épistémologie et celle de Gardin. Cette exagération et, plus généralement, les postures adoptées par les deux auteurs (obsidionale et distinctive pour Passeron; œcuménique et compréhensive pour Gardin) rappellent que non seulement les objets des sciences de l'homme, mais aussi les propositions qu'elles génèrent à propos de ces objets, sont situés historiquement et répondent à des enjeux parfois extérieurs à l'établissement de la connaissance scientifique. Après avoir analysé les implications du caractère historique de ces objets sur la

113. Grize 1994a, p. 288.

114. Proposition 3.4, scolie 1, Passeron 1991, p. 394.

115. Gardin 1993, p. 165.

116. Gardin 1995, p. 29. Similairement, Granger a défendu la complémentarité des différents types de modèles scientifiques en sciences de l'homme : Granger 1971, p. 44.

117. Gardin 2009, p. 174 
formalisation et la scientificité de ces sciences, nous examinerons la dispute de Passeron et Gardin à propos de l'historicité des pratiques de recherche.

\section{Le caractère historique des sciences de l'homme}

Les problèmes liés au caractère historique et à la détermination pragmatique des raisonnements en sciences de l'homme recouvrent trois aspects différents : la production de ces connaissances, leur réception, et l'anticipation de cette réception par les chercheurs. L'enjeu est de bien saisir l'opposition de Gardin et de Passeron sur la dé-historicisation induite par la formalisation, puis d'analyser leurs conceptions divergentes des processus du changement scientifique. Enfin, je montrerai que, paradoxalement, ce fut le tenant de l'approche la plus pragmatique sur le plan théorique qui, en pratique, adoptait une approche la moins susceptible de faciliter et de généraliser les applications et la diffusion de ses propositions.

\subsection{Formaliser implique-t-il de dé-historiciser?}

À un certain niveau d'analyse, tout processus d'abstraction (qu'il soit idéaltypique ou formel) suppose, à un certain degré, l'extraction et l'isolation du phénomène considéré par rapport à son contexte d'observation. Les problèmes épistémologiques soulevés à ce propos comprennent, premièrement, le fait de savoir si les sciences de l'homme possèdent à cet égard une spécificité; et, deuxièmement, la manière d'établir le degré adéquat de cette désindexation de l'objet par rapport à son contexte.

L'argument principal de Passeron en faveur d'une conception limitative de l'usage de la formalisation en sciences historiques reposait sur l'idée qu'un lien doit nécessairement être maintenu entre les concepts descriptifs et les réalités historiquement situées, passées et présentes, auxquels ces concepts font référence. Le sens de ces concepts historiques (tel que celui de féodalité) est dépendant de ces indexations. Rompre ce lien revient à les vider de leur sens. Les travaux de Gardin manquaient, selon lui, à cette nécessité.

Or, ce dernier opposait que la formalisation et la modélisation n'impliquent pas une telle dé-historicisation. Tout en admettant sans difficulté l'historicité des concepts employés dans les sciences de l'homme, Gardin précisait ainsi le but de l'« épistémologie pratique » qu'il défendait :

«[...] en partant des flottements observés dans les opérations constitutives de nos raisonnements, [elle s'emploie] à les réduire par la recherche systématique des conditions ou du contexte dans lesquels chacune de ces opérations est jugée recevable, à un moment donné. » ${ }^{118}$

Il insistait ainsi sur le caractère historiquement situé des opérations de connaissance menées dans les sciences de l'homme. Par ailleurs, lorsqu'il soulignait «l'inclusion nécessaire des données comparatives dans la base de faits [d'un système expert], au même titre que les données d'observation proprement dites » ${ }^{119}$, il admettait

118. Gardin 1987a, p. 244.

119. Gardin 1987a, p. 234. 
que les descriptions des phénomènes analysés dépendent de la série des descriptions antérieures qui donnent leur consistance aux concepts descriptifs.

Gardin défendait ici des positions similaires à celles de Granger. Pour ce dernier, admettre la richesse sémantique des langues naturelles, et leur dimension pragmatique, n'impliquait pas d'abandonner une épistémologie rationaliste au profit d'une herméneutique subjectiviste. Au début des années 1960, il posait ce problème dans les termes suivants :

"Tout objet scientifique est nécessairement constitué par l'opposition
d'une structure et d'un contenu vécu, lequel renvoie finalement à l'in-
dividuel. Mais dans le fait humain, l'individuation ne peut être indéfi-
niment neutralisée, comme il arrive dans d'autres domaines; il faut que
la science parvienne à saisir d'une certaine manière l'individuel en tant
que tel. Les modèles vers lesquels elle s'oriente permettent justement de
l'approcher. » ${ }^{120}$

Poursuivant le projet d'une «philosophie du style », Granger visait précisément à maintenir cette rigueur à ce propos, afin de ne pas abandonner à la seule littérature la saisie de la singularité. Les modèles des sciences humaines et, partant, le ou les langages avec lesquels ils sont établis, doivent alors nécessairement posséder certaines différences avec le langage ordinaire. Comme le notait Granger, deux aspects du caractère historique des sciences, la singularité des personnes et des activités, et le fait qu'elles évoluent, ne sont pas incompatibles avec l'objectif d'une science de l'homme. Ceci vaut également pour la dispute qui nous intéresse, laquelle s'inscrit dans les évolutions intellectuelles allant des années 1960 à la fin $\mathrm{du} \mathrm{xx}^{\mathrm{e}}$ siècle : il donc convient d'examiner la prise en compte par Gardin et par Passeron du caractère historique des pratiques scientifiques.

\subsection{Les mécanismes du changement scientifique}

Pour cela, j'évoquerai d'abord les conditions d'adoption de langages scientifiques pour traiter ensuite des modalités de décision entre théories concurrentes.

Passeron reconnaissait un rôle déterminant à l'une des caractéristiques des langages employés en sciences de l'homme : leur simplicité. Selon lui, la simplicité du langage dans lequel une théorie y est exprimée facilite sa reproduction et sa diffusion. Elle détermine aussi, par conséquent, son succès ${ }^{121}$. Il en découle que le succès d'un langage formalisé est peu probable, étant supposé que son coût d'apprentissage ne satisfait guère l'exigence d'une «facile reproduction »- du moins pour ce qui concerne les sciences de l'homme.

Selon Gardin, d'autres critères que les facteurs sociolinguistiques et les processus d'imitation doivent être considérés pour expliquer le succès ou l'échec d'une proposition théorique. Il contestait le fait de réduire la fonction des «langues spéciales » (c'est-à-dire spécifiques à un domaine scientifique) à la distinction et à la justification sociale de l'expertise ${ }^{122}$. S'il a pu ainsi reconnaître les effets de facteurs non épistémologiques pour l'intelligibilité de courtes séquences de l'histoire

\footnotetext{
120. Granger 1960, p. 316.

121. Passeron 1991, p. 363

122. Gardin 1993, p. 163.
} 
des sciences, il les déniait toutefois pour son évolution à plus long terme ${ }^{123}$. L'idée d'une différence des mécanismes actifs en fonction de l'échelle considérée de la dynamique des sciences considérées conduit au problème de la validation des théories scientifiques.

Des propositions théoriques peuvent être contradictoires entre elles, nécessitant donc de les départager pour n'en retenir qu'une seule. Pour ceci, divers critères de décision peuvent être employés : parcimonie, adéquation au réel, efficacité, etc. Rappelons que l'entreprise de Passeron était - comme le manifeste le sous-titre du Raisonnement sociologique - explicitement dirigée contre les thèses de Popper. Il s'opposait, en particulier, au principe de réfutation mis par Popper au centre de sa conception du changement scientifique (le choix d'une théorie étant fondé non sur sa validité intrinsèque, mais sur l'impossibilité actuelle de la réfuter). Selon Passeron, du fait, là aussi, de la dépendance constitutive des concepts historiques aux contextes auxquels ils réfèrent et à leurs contextes d'énonciation, il est impossible de réfuter une théorie en sciences historiques. Par conséquent, il est impossible de considérer ces théories à partir de l'épistémologie scientifique poppérienne.

Gardin identifiait une faiblesse dans cette thèse de Passeron. Il relevait que ce dernier a aussi suggéré qu'il existe certains critères permettant d'arbitrer entre des propositions différentes (différentes en raison de leur langue d'expression ou de leur « école de pensée », lesquelles peuvent outrepasser les frontières linguistiques) ${ }^{124}$. Selon Passeron, puisqu'en sciences historiques les énoncés formels doivent toujours être reformulés en langue naturelle, les errances potentielles de l'interprétation - les sur-, sous- et més- interprétations - doivent être contrôlées autrement que par une formalisation logico-mathématique. Or, comme le pointait Gardin, Passeron laissait ces critères de décision vagues et indéfinis, rendant la possibilité de décider plus programmatique qu'effective. En fait, Passeron renvoyait in fine la responsabilité de la définition de ces critères aux pairs et à la postérité :

«Dans les sciences sociales, la "valeur" d'un résultat ou d'une ouvre scientifique n'est finalement mesurable que par la trace paradigmatique qu'ils laissent dans la mémoire vive des communautés savantes capables de les réutiliser. Aucune docimologie comptable, aucune méthodologie fermée ne saurait évaluer cette valeur scientifique en lieu et place de la postérité savante. Et nul ne peut prétendre, en un lieu et à un moment de l'histoire des sciences sociales, avoir sur les connaissances qu'elles produisent le "regard naïf de la postérité". » ${ }^{125}$

Le terme «paradigmatique » doit certainement être compris ici au sens d' « exemple paradigmatique » (l'un des sens d'ailleurs attribué par Thomas Kuhn à ce terme); par docimologie, qui désigne en général la science des examens, des concours et de la notation scolaire, on doit probablement comprendre ici les procédures d'évaluation quantifiées de l'activité scientifique, c'est-à-dire ce que représente aujourd'hui la scientométrie dans ses usages gestionnaires ${ }^{126}$. Cette idée selon laquelle les théo-

123. Gardin 1999, p. 122.

124. Gardin s'appuyait sur Passeron 1991, p. 386 et 388. Ces passages sont inchangés dans la seconde édition du livre, p. 594 et 597.

125. Passeron 1991, p. 370.

126. Gingras 2014. 
ries scientifiques sont déterminées en dernier lieu par des facteurs sociologiques laissait Gardin profondément insatisfait ${ }^{127}$. Ceci, d'autant plus que Passeron tirait une autre implication, celle de l'impossibilité d'une cumulativité du savoir en sciences historiques : " [l]a sociologie n'a pas et ne peut prendre la forme d'un savoir cumulatif, c'est-à-dire d'un savoir dont un paradigme théorique organiserait les connaissances cumulées. ${ }^{128}$. Gardin contestait également cette thèse, opposant là aussi l'exemple de l'archéologie où l'on observe, selon lui, une progression cumulative des savoirs ${ }^{129}$.

Les deux auteurs se distinguent ainsi fondamentalement dans leurs conceptions de l'histoire des sciences (de l'homme). Des conceptions théoriques aussi divergentes laissent supposer des pratiques personnelles de recherche l'étant tout autant. Ce sera le dernier aspect que nous examinerons.

\subsection{Pragmatique de l'intervention théorique}

Analyser les conformités et les décalages entre les discours normatifs et les pratiques est un principe de méthode devenu banal en ethnologie et en sociologie. Dans le cas d'une dispute en sciences de l'homme, l'aspect normatif revient aux prescriptions épistémologiques et l'aspect pratique revient aux modalités communicationnelles des productions discursives. Il y a, dans le rapport entre ces deux aspects, une différence fondamentale dans les manières dont Gardin et Passeron ont abordé l'intervention théorique en sciences de l'homme. Là où le premier s'est attaché à optimiser la communication des propositions scientifiques par l'explicitation et la simplification, le second négligeait davantage les aspects pratiques et a adopté un mode d'exposition tel que la revendication de la spécificité épistémologique des sciences historiques s'accompagnait d'une constitution ésotérique de ces savoirs, à travers l'élévation du coût d'entrée causée par l'opacification délibérée du sens des énoncés.

Dans le chapitre «Les paradis artificiels du formalisme » du Raisonnement sociologique, Passeron admettait que la réception du programme logiciste fut minime en sociologie, tout en considérant qu'elle fut effective en archéologie du fait des travaux menés par Gardin. Malgré cette réception réduite, Passeron indiquait deux raisons de s'y opposer ${ }^{130}$. La première est conceptuelle : ce programme contrevenait (comme on l'a vu) à la claire distinction entre langage naturel et langage formel et à l'analyse de leurs rapports. La deuxième est d'ordre psychosociologique, à savoir, le «puissant » «fantasme de pureté logique » qu'il suscitait, au détriment des utilisateurs de langues naturelles. Faute d'enquête empirique sur la réception et sur les usages de ces propositions théoriques en sciences de l'homme, difficile d'évaluer les effets du fantasme mentionné par Passeron. Tout au plus, suggérons que l'anticipation spéculative des effets des différentes thèses opposées est un ressort très usité des disputes intellectuelles. Passeron s'est lui-même exprimé sur les vertus pédagogiques de l'exagération et celles, épistémologiques et politiques, du soupçon. Les premières consistent, selon la prescription d'Ignace de Loyola (qu'il

127. Gardin 1993, p. 164. Cette «aporie »a aussi été pointée - sans toutefois mentionner les remarques de Gardin - dans : Souloumiac et Fossier 2003, p. 100.

128. Proposition 2.1, Passeron 1991, p. 364 .

129. Plutniak 2017b, p. 15-17.

130. Passeron 1991, p. 154-155. 
invoque), à tordre un bâton à l'inverse de sa courbure initiale de manière à le remettre droit ${ }^{131}$. L'absence de prise en considération, par Passeron, des différences entre les projets successifs de Gardin et des précautions nuancées exprimées par ce dernier revenait-elle à une telle correction de perspective? Les spéculations de Passeron quant aux effets de la formalisation des énoncés doivent-elles alors être comprises comme une pratique du soupçon, c'est-à-dire, selon lui, la possibilité de formuler l' « hypothèse du pire » ${ }^{132}$ ? Cette manière de pratiquer l'anticipation n'est toutefois pas la seule envisageable : si Gardin a pu, lui aussi, se livrer à quelques exercices de ce genre, il le fit toutefois explicitement, en plaçant ses propositions sous l'intitulé "prospective ${ }^{133}$, tout en se dispensant prudemment de recourir aux vertus supposées de l'exagération.

Passeron assumait pleinement de publier une construction théorique sous une forme telle que sa réception, de même que le contrôle de son interprétation, supposent un effort important de la part du lecteur. Le pari d'une formalisation, parce qu'il le tenait pour illusoire, était abandonné au profit d'une expression en langage naturel dont la nécessaire indexicalité et la flexibilité sémantique étaient assumés. Le plein usage de la complexité syntaxique et sémantique du langage naturel était donc à la fois prescrit pour la pratique des sciences historiques et employé par l'auteur pour formuler une théorie de ces sciences. L'exercice réalisé par Passeron revenait à pousser ce principe à ses limites, en adoptant un style abstrait, dense, aux limites du conceptisme, supposant un effort particulièrement poussé de lecture. Paul Veyne considérait ainsi qu'il s'agissait d'un style «qui dit le droit fil conceptuel, mais au moyen de contournements, d'arabesques, de gongorismes, d'incises, de papillottements ${ }^{134}$. Passeron s'est prononcé sur le rapport quasi esthétique que lui procurait la « condensation des signifiants», plaisir qui prenait sa source dans ses lectures de Spinoza ${ }^{135}$. Jean-Louis Fabiani a souligné combien ce style ne peut se comprendre qu'en rapport avec «l'humeur philosophique » des années $1950^{136}$, une humeur dont le traducteur espagnol du Raisonnement sociologique, José Luis Moreno Pestaña, a décrit les principaux aspects ${ }^{137}$. Ce parti pris stylistique était toutefois adopté par Passeron au risque d'empêcher la transmission du sens des propositions ou, du moins, au risque de la limiter à une portion congrue du lectorat potentiel, celui ayant acquis les aptitudes, le goût et les dispositions nécessaires à effectuer une si exigeante lecture. En somme, il s'assurait ainsi une place parmi les auteurs ayant incarné une figure du théoricien des sciences de l'homme conçu comme producteur de discours complexes, potentiellement obscurs, et dont le coût d'entrée garantit à celui qui s'en acquitte d'intégrer le cercle étroit d'un lectorat sélectif.

Passeron n'ignorait évidemment pas les effets de ce type de style sur ses lecteurs. Il appelait «majoration stylistique » le processus de complexification par lequel un énoncé est reformulé à l'aide d'un autre lexique (éventuellement formalisé), sans que son sens en soit transformé :

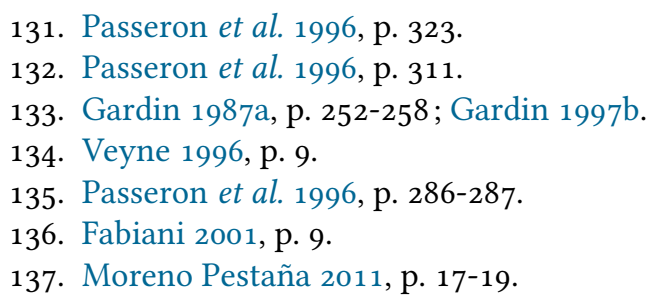


«Ce n'est pas trop grave, même si c'est fatiguant pour le lecteur, qui est toujours en droit de n'accepter de payer cette surprime de réception que si le contenu assertorique qu'il achète ainsi, à un prix majoré par le détenteur d'un monopole théorique, en vaut la peine [...] ${ }^{138}$

Ajoutons que la remarque vaut tout autant pour des énoncés dont les reformulations génèrent une plus-value d'intelligibilité : la surprime de réception n'en est pas moins fatigante. Les traces matérielles d'une telle exaspération intellectuelle peuvent être observées sur une copie du Raisonnement sociologique : celle à partir de laquelle Gardin rédigea sa note critique de 1993, dans les marges de laquelle il s'exclama avec lassitude «Quel jargon! » ${ }^{139}$.

\section{Irrésolution}

Cet article a proposé une contribution à l'histoire conceptuelle du structuralisme et de sa réception. La méthode adoptée a consisté à analyser la dispute épistémologique entre des auteurs ayant eu en commun d'établir des positions à distances raisonnables de plusieurs formes de structuralisme, dans un contexte intellectuel qualifié de poststructuraliste et marqué par un rejet catégorique de ces structuralismes. Les propositions de ces auteurs, fondées d'une part sur une critique approfondie de ces approches et, d'autre part, sur le maintien de valeurs épistémiques réalistes et rationalistes, s'intègrent difficilement dans un schéma « générationnel » binaire selon lequel se succéderaient et s'opposeraient une période et un corpus structuraliste à une période et à un corpus poststructuraliste.

En nous concentrant sur la dispute entre Gardin et Passeron, il était attendu, qu'en dépit de ce point commun de leurs parcours intellectuels, ces deux auteurs se soient reconnus de fortes divergences. En effet, nous avons mis en évidence d'importants clivages entre leurs positions, tel qu'à propos de l'unité d'analyse dans l'étude des raisonnements (envisagés soit comme des propositions soit comme des énoncés) ou, encore, à propos des implications du caractère historique de l'objet et des productions des sciences de l'homme. De manière plus inattendue, nous avons également identifié : des différences peu considérées par les deux auteurs dans leur dispute, tels que les effets des modes d'exposition des constructions scientifiques sur leur réception; des différences exagérées par les auteurs, lesquelles doivent, au contraire, être nuancées, comme à propos de leurs conceptions respectives de la formalisation; enfin, des similarités négligées par les deux auteurs, notamment à propos du traitement moindre des aspects ontologiques.

Si ces résultats ont pour effet de contrarier les éventuelles revendications d'affiliation qu'autoriserait une lecture superficielle et dualiste entre structuralisme et poststructuralisme, ils permettent aussi d'identifier et de réaffirmer des orientations épistémologiques particulièrement utiles pour la pratique contemporaine des sciences de l'homme, dans un contexte où elles se trouvent directement menacées - à des degrés divers et que ce soit en Turquie, en Hongrie, ou en France notamment sur la base de leur discrédit scientifique.

138. Passeron 1991, p. 394.

139. Feuillet 1, Dossier « Troisième voie - narrativité », BNU. 


\section{Remerciements}

Cet article prolonge deux conférences, l'une «Archéologie, formalisation et sciences humaines : autour du débat Passeron-Gardin » présentée à Toulouse en 2015 et l'autre "Rationalist criticisms of structuralism in the "poststructuralist" period. Formalization and linguistic issues in the debates between 7.-C. Gardin, 7.-C. Passeron and G.-G. Granger » donnée à l'université de São Paulo en 2018. Je remercie Sophie Assal (archives Claude Lévi-Strauss du Laboratoire d'anthropologie sociale); Denise Bally (archives de l'EHEss à Marseille); Aurélie Montagne-Bôrras (archives de la Maison Archéologie \& Ethnologie, Nanterre); ainsi que Michaël Gasperoni, Bertrand Augier, Jérôme Lamy, Arnaud Saint-Martin, et les évaluateurs, Bruno Ambroise et Volny Fages, pour leurs précieux commentaires sur ce texte, qui n'engage néanmoins que son auteur.

\section{Références}

Abbott, Andrew (2001), "Chaos of Disciplines », dans Chaos of Disciplines, Chicago (Ill.) : University of Chicago Press, p. 3-33, ISBN : 0-226-00100-8.

Angermuller, Johannes (2007), «Qu'est-ce que le poststructuralisme français? À propos de la notion de discours d'un pays à l'autre », Langage et société, 120, 2, p. 17-34, DOI : $10.3917 /$ s. 120.0017.

Bert, Jean-François et Jérôme Lamy (éd.) (2016), Résonances des structuralismes, Paris : Éditions des archives contemporaines, ISBN : 9-782813-002433.

Bourdieu, Pierre, Jean-Claude Passeron et Jean-Claude Chamboredon (1969), Le Métier de sociologue, Paris : Mouton.

Brandmayr, Federico (2018), "Hétérogénéité et flexibilité dans les usages politiques de l'argument de l'“excuse sociologique” », Zilsel, 4, p. 59-91, DOI : 10.3917/zil.004.0059.

Bronner, Gérald et Étienne Géhin (2017), Le danger sociologique, Paris : Presses universitaires de France, ISBN : 978-2-13-075024-6.

Cahuc, Pierre et André Zylberberg (2017), Le négationnisme économique. Et comment s'en débarrasser, Paris : Flammarion, ISBN : 978-2-08-139599-2.

Chateauraynaud, Francis (2003), Prospéro. Une technologie littéraire pour les sciences humaines, Paris : CNRS Éditions, ISBN : 2-271-06133-4.

Costey, Paul et Arnaud Fossier (2009), «Entretien avec Jean-Claude Passeron », Tracés. Revue de sciences humaines, 4, p. 127-144, DOI : 10.4000/traces. 3983.

Dosse, François (1991), Histoire du structuralisme. I. Le champ du signe, 1945-1966, Paris : La Découverte, ISBN : 2-7071-2103-7.

Dosse, François (1992), Histoire du structuralisme. II. Le chant du cygne. 1967 à nos jours, Paris : La Découverte, ISBN : 978-2-7071-7461-1.

Fabiani, Jean-Louis (2001), « Avant-propos », dans Le goût de l'enquête. Pour Jean-Claude Passeron, sous la dir. de Jean-Louis Fabiani, Paris : L'Harmattan, p. 7-11, ISBN : 2-7475-0639-8.

Fabiani, Jean-Louis (2007), «Disputes, polémiques et controverses dans les mondes intellectuels. Vers une sociologie historique des formes de débat 
agonistique », Mil neuf cent. Revue d'histoire intellectuelle, 25, $1:$ Les formes de la controverse, p. 45-60.

Fabiani, Jean-Louis (2016), Pierre Bourdieu. Un structuralisme héroïque, Paris : Éditions du Seuil, ISBN : 978-2021290325.

Gardin, Jean-Claude (1965), « Analyse documentaire et analyse structurale en archéologie », L'Arc, 26, p. 64-68.

Gardin, Jean-Claude (1971), «Archéologie et calculateurs : nouvelles perspectives », Revue internationale des sciences sociales, 23, 2, p. 204-218.

Gardin, Jean-Claude (1973), « Document Analysis and Linguistic Theory », fournal of Documentation, 29, 2, p. 137-168, DOI : 10.1108/eb026553.

Gardin, Jean-Claude (1974a), « Analyse documentaire et analyse structurale en archéologie », Langages, 8, 35, p. 82-86, DOI : 10.3406/lgge.1974.2267.

Gardin, Jean-Claude (1974b), « Analyse documentaire et théorie linguistique », dans Les analyses de discours, Neuchâtel : Delachaux et Niestlé, p. 120-168.

Gardin, Jean-Claude (1974c), « Des sciences du discours au discours de la science », dans Les analyses de discours, Neuchâtel : Delachaux et Niestlé, p. $10-60$.

Gardin, Jean-Claude (1979), Une archéologie théorique, Paris : Hachette, ISBN : 2-01-005627-2.

Gardin, Jean-Claude (1987a), «Épilogue », dans Jean-Claude Gardin, Olivier Guillaume, Peter Q. Herman, Antoinette Hesnard, Marie-Salomé Lagrange, Monique Renaud et Élizabeth Zadora-Rio, Systèmes experts et sciences humaines. Le cas de l'archéologie, Paris : Eyrolles, p. 233-255.

Gardin, Jean-Claude (1987b), «From Discourse Analysis to the Use of Expert Systems in the Sciences of Man », Communication and Cognition, 4, 3, p. 189-201.

Gardin, Jean-Claude (1987c), « La logique, naturelle ou autre, dans les constructions de sciences humaines », Revue européenne des sciences sociales, 25, 77, p. 179-195.

Gardin, Jean-Claude (1988), «Une contribution des humanités à l'informatique », dans L'histoire de l'informatique en France, Colloque sur l'histoire de l'informatique en France, sous la dir. de Philippe Chatelin, Grenoble : Institut national polytechnique de Grenoble, t. 2, p. 135-154, ISBN : 2-9502887-0-7.

Gardin, Jean-Claude (1991a), Le calcul et la raison. Essais de formalisation du discours savant, 46, Paris : Éditions de l'École des hautes études en sciences sociales.

Gardin, Jean-Claude (1991b), «Le rôle du sujet dans les sciences de l'homme : essais d'évaluation objective », Revue européenne des sciences sociales, 29, 89, p. 91-102.

Gardin, Jean-Claude (1993), «Les embarras du naturel », Archives Européennes de Sociologie / European fournal of Sociology / Europäisches Archiv für Soziologie, 34, 1, p. 152-165, DOI : 10.1017/S0003975600006603.

Gardin, Jean-Claude (1995), «Calcul, informatique et raisonnement en archéologie », dans Raisonnement et calcul, sous la dir. de Denis Miéville, 63, Neuchâtel : Université de Neuchâtel, p. 11-33.

Gardin, Jean-Claude (1997a), «Autocritique d'un texte archéologique : logique naturelle et logique de champs », dans Logique, discours et pensée. Mélanges 
offerts à Jean-Blaise Grize, sous la dir. de Denis Miéville et

Alain Berrendonner, 52, Berne : Peter Lang, p. 37-57, ISBN : 3-906759-18-o.

Gardin, Jean-Claude (1997b), « Le questionnement logiciste et les conflits d'interprétation », Enquête, 5, p. 35-54, DOI : 10.4000/enquete.1043.

Gardin, Jean-Claude (1999), « Archéologie, formalisation et sciences sociales », Sociologie et sociétés, 31, 1, p. 119-127, DOI : 10.7202/001282ar.

Gardin, Jean-Claude (2001), «Entre modèle et récit : les flottements de la troisième voie », dans Le modèle et le récit, sous la dir. de Jean-Yves Grenier, Claude Grignon et Pierre-Michel Menger, Paris : Éditions de la Maison des sciences de l'homme, p. 457-487, ISBN : 2-7351-0905-4.

Gardin, Jean-Claude (2009), «Sciences de l'archéologie et sciences de la nature : entreprises novatrices depuis trente ans », dans Sciences de l'homme et sciences de la nature. Essais d'épistémologie comparée, sous la dir. de Claude Grignon et Claude Kordon, Paris : Éditions de la Maison des sciences de l'homme, p. 167-189, ISBN : 978-2-7351-1243-2.

Gingras, Yves (2014), Les dérives de l'évaluation de la recherche. Du bon usage de la bibliométrie, Paris : Raisons d'agir éditions, ISBN : 978-2-91210-77-56.

Granger, Gilles-Gaston (1960), Pensée formelle et sciences de l'homme, 2, Paris : Aubier-Montaigne.

Granger, Gilles-Gaston (1967), Pensée formelle et sciences de l'homme, 2e éd., 2, Paris : Aubier-Montaigne.

Granger, Gilles-Gaston (1969), «Propositions pour un positivisme », Man and World, 2, 3, p. 386-409.

Granger, Gilles-Gaston (1971), «L'explication dans les sciences sociales », Social Science Information, 10, 2, p. 31-44, DOI : $10.1177 / 053901847101000202$.

Granger, Gilles-Gaston (1982a), « À quoi servent les noms propres? », Langages, 66, p. 21-36.

Granger, Gilles-Gaston (1982b), « Modèles qualitatifs, modèles quantitatifs dans la connaissance scientifique », Sociologie et sociétés, 14, 1, p. 5-12, DOI : 10.7202/006768ar.

Granger, Gilles-Gaston (1989), « Les conditions proto-logiques des langues naturelles », Philosophiques, 16, 2, p. 245-256, DOI : 10.7202/027080ar.

Grignon, Claude (2001), «La formalisation et les sciences du récit », dans Le modèle et le récit, sous la dir. de Jean-Yves Grenier, Claude Grignon et Pierre-Michel Menger, Paris : Éditions de la Maison des sciences de l'homme, p. 7-43, ISBN : 2-7351-0905-4.

Grize, Jean-Blaise (1993), «Logique naturelle et représentations sociales », Papers on Social Representations, 2, 3, p. 151-159.

Grize, Jean-Blaise (1994a), «Logique naturelle et sociologie », L'Année sociologique, 44, p. 281-289, DOI : 10.2307/27889376.

Grize, Jean-Blaise (éd.) (1994b), Sémiologie du raisonnement, 9, Berne, Francfort-sur-le-Main et New York : Peter Lang, ISBN : 978-3261034823.

Grize, Jean-Blaise (1995), «Le raisonnement est-il un pis aller?», dans Raisonnement et calcul, sous la dir. de Denis Miéville, 63, Neuchâtel : Centre de recherches sémiologiques, p. 1-10.

Grize, Jean-Blaise (1996), Logique naturelle et communications, Paris : Presses universitaires de France. 
Grize, Jean-Blaise (2000), « Les discours du savoir. Pour un dialogue avec Jean-Claude Gardin », Revue européenne des sciences sociales, 38, 119, p. 131-138, DOI : 10.4000/ress. 677.

Joly, Marc (2018), Pour Bourdieu, Paris : Éditions du CNRS, ISBN : 978-2271118677.

Lacour, Philippe (2005), « Gilles-Gaston Granger et la critique de la raison symbolique », Texto!

Lahire, Bernard (2016), Pour la sociologie. Et pour en finir avec une prétendue «culture de l'excuse », Paris : La Découverte, ISBN : 9782707188601.

Lemieux, Cyril (éd.) (2017), Pour les sciences sociales. 101 livres, Paris : Éditions de l'École des hautes études en sciences sociales, ISBN : 978-2-7132-2722-6.

Lenclud, Gérard (2001), «Le formel et le vécu », dans Le modèle et le récit, sous la dir. de Jean-Yves Grenier, Claude Grignon et Pierre-Michel Menger, Paris : Éditions de la Maison des sciences de l'homme, p. 425-456, IsBN : 2-7351-0905-4.

Léon, Jacqueline (2013), « Historiographie du structuralisme généralisé. Étude comparative », Les dossiers de HEL, 3.

Lepenies, Wolf (1990), Les trois cultures. Entre science et littérature, l'avènement de la sociologie, Paris : Éditions de la Maison des sciences de l'homme, ISBN : 2-7351-0344-7.

Mandelbaum, Maurice (1965), « The History of Ideas, Intellectual History, and the History of Philosophy », History and Theory, 5, p. 33-66, DOI : $10.2307 / 2504118$.

Milner, Jean-Claude (2002), Le périple structural. Figures et paradigme, Paris : Édition du Seuil, ISBN : 2-02-042792-3.

Mongin, Philippe (2003), "L'axiomatisation et les théories économiques », Revue économique, 54, 1, p. 99-138, DOI : 10.3917/reco.541.0099.

Moreno Pestaña, José Luis (2011), «Una filosofía de las ciencias históricas: presentación de la obra de Jean-Claude Passeron », dans El razonamiento sociológico. El espacio comparativo de las pruebas históricas, Madrid : Siglo XXI, p. 9-49, ISBN : 978-84-323-1444-5.

Passeron, Jean-Claude (1980), Les mots de la sociologie, thèse de doctorat d'État, Nantes : Université de Nantes.

Passeron, Jean-Claude (1991), Le raisonnement sociologique. L'espace non-poppérien du raisonnement naturel, Paris : Nathan, ISBN : 2-09-190412-0.

Passeron, Jean-Claude (2001a), «Formalisation, rationalité et histoire », dans Le modèle et le récit, sous la dir. de Jean-Yves Grenier, Claude Grignon et Pierre-Michel Menger, Paris : Éditions de la Maison des sciences de l'homme, p. 215-282, ISBN : 2-7351-0905-4.

Passeron, Jean-Claude (2001b), «La forme des preuves dans les sciences historiques », Revue européenne des sciences sociales, 39, 120, p. 31-76.

Passeron, Jean-Claude (2002), «Logique formelle, schématique et rhétorique », dans Argumentation. Preuve et persuasion, sous la dir. de Michel de Fornel et Jean-Claude Passeron, 2, Paris : Éditions de l'École des hautes études en sciences sociales, p. 149-181, ISBN : 2-7132-1423-8.

Passeron, Jean-Claude (2006), Le raisonnement sociologique. Un espace non poppérien de l'argumentation, Paris : Albin Michel, ISBN : 2-226-15889-8. 
Passeron, Jean-Claude, Raymonde Moulin et Paul Veyne (1996), «Entretien avec Jean-Claude Passeron. Un itinéraire de sociologue », Revue européenne des sciences sociales, 34, 103 : Pavane pour Fean-Claude Passeron, p. 275-354.

Piaget, Jean (1968), Le structuralisme, 1311, Paris : Presses universitaires de France.

Plutniak, Sébastien (2017a), «L'innovation méthodologique, entre bifurcation personnelle et formation des disciplines : les entrées en archéologie de Georges Laplace et de Jean-Claude Gardin », Revue d'histoire des sciences humaines, 31, p. 113-139, DOI : 10.4000/rhsh. 435.

Plutniak, Sébastien $(2017 \mathrm{~b})$, « Une contribution archéologique à la théorie des sciences sociales est-elle possible? Aspects de la controverse entre Jean-Claude Passeron et Jean-Claude Gardin », Palethnologie, 9, p. 7-21, DOI : 10.4000/palethnologie. 279.

Plutniak, Sébastien (2018), « Aux prémices des humanités numériques? La première analyse automatisée d'un réseau économique ancien (Gardin \& Garelli, 1961). Réalisation, conceptualisation, réception », ARCS. Analyse de réseaux pour les sciences sociales, 1.

Plutniak, Sébastien (2019), «À distances raisonnables des structuralismes : logique, langage, formalisation et sciences de l'homme. Une dispute du zoe siècle finissant », Zilsel. Science, technique, société, 6, p. 70-115, DOI : 10.3917/zil.006.0070.

Puech, Christian (éd.) (2013), Dossiers HEL 3.

Roux, Sophie (2010), «Forms of Mathematization (14th-17th Centuries) », Early Science and Medicine, 15, p. 319-337.

Russell, Bertrand (1905), « On Denoting », Mind, 14, 56, p. 479-493, DOI : $10.2307 / 2248381$.

Russell, Bertrand (1917), «On the Notion of Cause », dans Mysticism and Logic. And other Essays, London : George Allen \& Unwin, p. 180-208.

Russell, Bertrand (2006), «Sur la notion de cause », Philosophie, 89, 2, p. 3-20, DOI : $10.3917 /$ philo.089.0003.

Sapiro, Gisèle et Lucile Dumont (2016), « La diffusion internationale du structuralisme : entre appropriation et rejet », dans Résonances des structuralismes, sous la dir. de Jean-François Bert et Jérôme Lamy, Paris : Éditions des archives contemporaines, p. 123-138, ISBN : 9-782813-002433.

Skinner, Quentin (1969), « Meaning and Understanding in the History of Ideas », History and Theory, 8, 1, p. 3-53, DOI : 10.2307/2504188.

Souloumiac, Julien et Arnaud Fossier (2003), «Passeron : entre Weber et Wittgenstein », Tracés, 4, p. 83-102.

Stoczkowski, Wiktor (2001), «Raison narrative : des vertus cognitives du récit comparées à celles du modèle », Social Science Information, 40, 3, p. 347-371, DOI : $10.1177 / 053901801040003001$.

Toulmin, Stephen E. (1958), The Uses of Argument, Cambridge : Cambridge University Press.

Varenne, Franck (2008), « Modèles et simulations : pluriformaliser, simuler, remathématiser », Matière première, 1, 3, p. 153-180. 
Veyne, Paul (1996), «"Celui-Là n’a pas un bon cœur, que la gratitude fatigue” : Pour notre ami Jean-Claude Passeron », Revue européenne des sciences sociales, 34, 103 : Pavane pour fean-Claude Passeron, p. 5-14. 